\title{
Generalized Rough Fuzzy Ideals in Quantales
}

\author{
Saqib Mazher Qurashi $\mathbb{D}^{1,2}$ and Muhammad Shabir ${ }^{2}$ \\ ${ }^{1}$ Department of Mathematics, Government College University, Faisalabad, Pakistan \\ ${ }^{2}$ Department of Mathematics, Quaid-i-Azam University, Islamabad, Pakistan \\ Correspondence should be addressed to Saqib Mazher Qurashi; saqibmazhar@gcuf.edu.pk
}

Received 7 September 2017; Revised 19 November 2017; Accepted 10 December 2017; Published 16 January 2018

Academic Editor: Rigoberto Medina

Copyright (c) 2018 Saqib Mazher Qurashi and Muhammad Shabir. This is an open access article distributed under the Creative Commons Attribution License, which permits unrestricted use, distribution, and reproduction in any medium, provided the original work is properly cited.

\begin{abstract}
The paper examines the generalized rough fuzzy ideals of quantales. There are some intrinsic relations between fuzzy prime (primary) ideals of quantales and generalized rough fuzzy prime (primary) ideals of quantales. Homomorphic images of "generalized rough ideals, generalized rough prime (primary) ideals, and generalized rough fuzzy prime (primary) ideals" which are incited by quantale homomorphism are examined.
\end{abstract}

\section{Introduction}

The idea of "theory of rough sets" proposed by Pawlak $[1,2]$ to manage uncertainty and granularity in the information system has attracted the concern and attention of scientists and experts in different fields of science and technology. Late years have seen its wide applications in algebraic systems, knowledge discovery, data mining, expert systems, pattern recognition, granular computing, graph theory, machine learning, partially ordered sets, and so forth [3-15]. It is noted that the significant concepts in the classical theory of rough set are the lower and upper approximations obtained from equivalence relation on a universal set. In many cases, as pointed out by numerous researchers, the implementation of theory of rough set becomes restrictive if we use the condition of the equivalence relation in the model of Pawlak rough set. To get control of this issue, several authors generalized the classical rough set theory by using more general binary relations [16-20] or by employing coverings [21, 22]. Besides, theory of rough sets can also be generalized to the fuzzy environment by employing the notion of fuzzy sets of Zadeh [23], and the resulting notions are called fuzzy rough sets [24-27].

Recently, researchers have connected the ideas and techniques for rough set hypothesis to different algebraic structures. Biswas and Nanda [4] took a group as a ground set and presented the notions of rough groups and rough subgroups. Kuroki and Mordeson [28] discussed the structure of rough sets and rough groups. At that point in [29], Kuroki presented the thought of rough ideals in semigroup. Rough prime ideals and rough fuzzy prime ideals in semigroups were proposed by Xiao and Zhang [17]. Davvaz [30] gave the concepts of rough ideals in rings. He also wrote a short note on algebraic $T$ rough sets [31]. Kazanc1 and Davvaz in [32] gave rough prime (primary) ideals and rough fuzzy prime (primary) ideals in commutative rings. To overcome the confinement of equivalence relations in the process of establishing rough sets in a ring, Yamak et al. [19] introduced the concept of set-valued mappings as the basis of the generalized upper and lower approximations of a ring with the help of an ideal. Roughness in modules was researched by Davvaz and Mahdavipour [33]. Rasouli and Davvaz [14] presented the notion of rough ideals in MV-algebra. In BCK-algebras, rough ideals were defined by Jun [34]. Classical approximation theory has also been applied to some partially ordered structures. For instance, in [10], Estaji et al. investigated the concepts of upper and lower rough ideals in a lattice by introducing the relationships between lattice theory and rough sets. Zhan et al. discussed "a new rough set theory: rough soft hemirings" in [35]. Ma et al. gave the "applications of a kind of novel $Z$-soft fuzzy rough ideals to hemirings" and investigated "a survey of decision making methods based on certain hybrid soft set models" $[36,37]$.

The combination of rough set theory to soft set theory is very important. Feng et al. proposed rough soft sets by 
combining Pawlak rough sets and soft sets. In particular, Feng et al. put forth a novel concept of soft rough fuzzy sets by combining rough sets, soft sets, and fuzzy sets and we call it Feng-soft rough fuzzy set [38]. And in 2011, Meng et al. further discussed the Feng-soft rough fuzzy sets and put forward another kind of soft rough fuzzy sets, which is called Meng-soft rough fuzzy set [39]. These sets are limited and have a rigorous restrictive condition. Based on the above reason, Zhan and Zhu provided a novel concept of soft rough fuzzy sets, which is called a $Z$-soft rough fuzzy set [40]. As reported in [41, 42], characterizations of two kinds of hemirings based on probability spaces and reviews on decision making methods based on (fuzzy) soft sets and rough soft sets are discussed, respectively.

The structure of quantale was proposed by Mulvey [43] to study the spectrum of $C^{*}$-algebras. The idea of ideals (prime, primary) of quantale was given by Wang and Zhao $[44,45]$. Xiao and $\mathrm{Li}[16]$ generalized the ideals of quantale by means of set-valued mappings. The start of theory of rough sets for applying in algebraic structures, for example, semigroups, rings, modules, and groups, has been focused on a congruence relation. However, we obtain the restricted applications by using the congruence relation. To take care of this issue, Davvaz $[19,31]$ introduced the idea of a set-valued homomorphism for rings and groups. In this paper, we intend to generalize the results which have been proved in [46].

The arrangement of the paper is as per the following. In Section 2, we review some principal properties of rough sets, rough fuzzy sets, and ideals of quantale. In Section 3, we have introduced "generalized rough fuzzy ideal" and "generalized rough fuzzy prime (semiprime, primary) ideals" of quantales and give a few properties of such ideals. In Section 4, we will describe the images of generalized rough ideals and discuss how they are related. We will explain the relation between lower (upper) generalized rough and lower (upper) generalized approximations of their homomorphic images by using quantale homomorphism and set-valued homomorphism of quantale. In Section 5, we will discuss generalized rough fuzzy prime (primary) ideals based on quantale homomorphism. At last, the conclusion is given in Section 6.

\section{Preliminaries}

Here, we review a few ideas and results which will be vital in the following.

Definition 1 (see [2]). Let $(U, \sigma)$ be an approximation space, where $U$ is a nonempty set, and let $\sigma$ be an equivalence relation on $U$. For $x \in U$, the equivalence class of $x$, containing $x$, is denoted by $[x]_{\sigma}$. For $A \subseteq U$, the upper and lower approximations of $A$ are, respectively, defined as $\bar{\sigma}(A)=\left\{x \in U \mid[x]_{\sigma} \cap A \neq \emptyset\right\}, \underline{\sigma}(A)=\left\{x \in U \mid[x]_{\sigma} \subseteq A\right\}$. It is easy to verify that $\sigma(A) \subseteq A \subseteq \bar{\sigma}(A)$ for all $A \subseteq U$.

For more details on rough sets, rough fuzzy sets, and fuzzy rough sets, we refer to $[2,24,26,27]$. Throughout this paper, we shall use $Q_{t}$ and $Q_{t}^{\prime}$ for quantales, unless stated otherwise.
Definition 2 (see [47]). A complete lattice $Q_{t}$ having associative binary operation $*$ is called a quantale if it satisfies

$$
\begin{aligned}
& y *\left(\bigvee_{i \in I} z_{i}\right)=\bigvee_{i \in I}\left(y * z_{i}\right) ;\left(\bigvee_{i \in I} y_{i}\right) * z=\bigvee_{i \in I}\left(y_{i} * z\right), \\
& \text { for all } y, z, y_{i}, z_{i} \in Q_{t}(i \in I) .
\end{aligned}
$$

We will represent the top element of $Q_{t}$ by 1 and the bottom element by 0 throughout the paper. Let $A, B \subseteq Q_{t}$, and we define $A * B$ by the set $\{x * y \mid x \in A, y \in B\}, A \vee B$ by $\{x \vee y \mid x \in A, y \in B\}$ and $\bigvee_{i \in I} A_{i}=\left\{\bigvee_{i \in I} x_{i} \mid x_{i} \in A_{i}\right\}$.

Definition 3 (see [44]). Let $Q_{t}$ be a quantale. A nonempty subset $A$ of $Q_{t}$ is said to be an ideal of $Q_{t}$ if the following conditions hold:

(1) For all $z_{1}, z_{2} \in A, z_{1} \vee z_{2} \in A$ is implied.

(2) If $z \in Q_{t}, z^{\prime} \in A$ and $z \leqslant z^{\prime}$ imply $z \in A$.

(3) For all $z \in Q_{t}$ and $z^{\prime} \in A$, then $z * z^{\prime} \in A$ and $z^{\prime} * z \epsilon$ A.

An ideal $A$ is said to be a prime ideal if $z * z^{\prime} \in A$ implies $z \in A$ or $z^{\prime} \in \mathrm{A}$ for all $z, z^{\prime} \in Q_{t}$.

An ideal $A$ is said to be a semiprime ideal if $z * z \in A$ implies $z \in A$ for all $z \in Q_{t}$.

Primary ideal is an ideal $A$ of $Q_{t}$ if for all $x, z \in Q_{t}, x * z \epsilon$ $A$ and $x \notin A$ imply $z^{n} \in A$ for some positive integer $n$, where $z^{n}=\underbrace{z * \cdots * z}_{n}$.

As it is well known in the fuzzy theory established by Zadeh [23], a fuzzy subset $g$ of $Q_{t}$ is defined as a map from $Q_{t}$ to the unit interval $[0,1]$. The symbols $\wedge$ and $\vee$ will denote the respective infimum and supremum.

Definition 4 (see [24]). Let $(W, \sigma)$ be an approximation space. A fuzzy subset $g$ is a mapping from $W$ to $[0,1]$, then for $x \in$ $W$, one defines

$$
\begin{aligned}
& \underline{\sigma}(g)(z)=\bigwedge_{p \in[z]_{\sigma}} g(p) ; \\
& \bar{\sigma}(g)(z)=\bigvee_{p \in[z]_{\sigma}} g(p) .
\end{aligned}
$$

They are called the lower and upper approximations of $g$, respectively. If $\underline{\sigma}(g) \neq \bar{\sigma}(g)$, then $\sigma(g)=(\underline{\sigma}(g), \bar{\sigma}(g))$ is called a rough fuzzy set with respect to $\sigma$. For $\alpha \in[0,1]$, the sets

$$
\begin{aligned}
g_{\alpha} & =\{x \in W \mid g(x) \geq \alpha\} ; \\
g_{\alpha^{+}} & =\{x \in W \mid g(x)>\alpha\}
\end{aligned}
$$

are called $\alpha$-cut and strong $\alpha$-cut of the fuzzy set $g$, respectively.

Definition 5 (see [46]). A nonempty fuzzy subset $g$ of $Q_{t}$ is called a fuzzy ideal of $Q_{t}$, if the following conditions are satisfied:

(1) If $c \leq d$, then $g(d) \leq g(c)$.

(2) $g(c) \wedge g(d) \leq g(c \vee d)$.

(3) $g(c) \vee g(d) \leq g(c * d)$. 
From (1) and (2) in Definition 5, it is observed that $g(c \vee$ $d)=g(c) \wedge g(d)$ for all $c, d \in Q_{t}$. Thus, a fuzzy set $g$ is a fuzzy ideal of $Q_{t}$ if and only if $g(c \vee d)=g(c) \wedge g(d)$ and $g(c * d) \geq g(c) \vee g(d)$ for all $c, d \in Q_{t}$.

Definition 6 (see [46]). A nonconstant fuzzy ideal $g$ of a quantale $Q_{t}$ is called a fuzzy prime ideal of $Q_{t}$ if for all $c, d \in$ $Q_{t}$,

$$
g(c * d)=g(c) \text { or } g(c * d)=g(d) .
$$

Note that we require a fuzzy prime ideal of a quantale to be a nonconstant in order to keep consistent with the definition of prime ideals of quantales [45]. Therefore, throughout this paper, a fuzzy ideal of a quantale is always assumed to be nonconstant. For fuzzy semiprime and fuzzy primary ideals, see [46].

Proposition 7 (see [46]). Let $g$ be a fuzzy subset of a quantale $Q_{t}$. Then $g$ is a fuzzy (prime, semiprime, primary) ideal of $Q_{t}$ if and only if for each $\alpha \in[0,1], g_{\alpha}$ (resp., $\left.g_{\alpha^{+}}\right)$is either empty or (prime, semiprime, primary) ideal of $Q_{t}$.

Throughout this paper, $f$-ideal, $f$-prime, $f$-semiprime, and $f$-primary ideals will denote fuzzy ideal, fuzzy prime, fuzzy semiprime, and fuzzy primary ideals of quantales, unless stated otherwise. We use $\mathscr{F}\left(Q_{t}\right)$ to denote the set of all fuzzy subsets of $Q_{t}$.

The concept of generalized rough sets is a generalization of Pawlak's rough set. In rough set theory, an equivalence relation is the basic requirement for lower and upper approximations. Sometimes it is difficult to find such an equivalence relation among the elements of the set under investigation. In such situations, generalized rough set approach can be useful.

Definition 8 (see [19]). Let $U$ and $W$ be two nonempty universes. Let $H$ be a set-valued mapping given by $H: U \rightarrow$ $P(W)$, where $P(W)$ is the power set of $W$. Then the triple $(U, W, H)$ is referred to as a generalized approximation space or generalized rough set. Any set-valued function from $U$ to $P(W)$ defines a binary relation from $U$ to $W$ by setting $\sigma_{H}=$ $\{(x, y) \mid y \in H(x)\}$. Obviously, if $\sigma$ is an arbitrary relation from $U$ to $W$, then a set-valued mapping $H_{\sigma}: U \rightarrow P(W)$ can be defined by $H_{\sigma}(x)=\{y \in W \mid(x, y) \in \sigma\}$, where $x \in U$. For any set $A \subseteq W$, the lower and upper approximations represented by $\underline{H}(A)$ and $\bar{H}(A)$, respectively, are defined as

$$
\begin{aligned}
& \underline{H}(A)=\{z \in U \mid H(z) \subseteq A\}, \\
& \bar{H}(A)=\{z \in U \mid H(z) \cap A \neq \emptyset\} .
\end{aligned}
$$

We call the pair $(\underline{H}(A), \bar{H}(A))$ generalized rough set, and $\underline{H}, \bar{H}$ are termed as lower and upper generalized approximation operators, respectively.

If $W=U$ and $\sigma_{H}=\{(x, y) \mid y \in H(x)\}$ is an equivalence relation on $U$, then the pair $\left(U, \sigma_{H}\right)$ is the Pawlak approximation space. Therefore, a generalized rough set is an extended notion of Pawlak's rough set [16].
Definition 9 (see [16]). Let $\left(Q_{t}, *_{1}\right)$ and $\left(Q_{t}^{\prime}, *_{2}\right)$ be two quantales. A set-valued mapping $H: Q_{t} \rightarrow P^{*}\left(Q_{t}^{\prime}\right)$, where $P^{*}\left(Q_{t}^{\prime}\right)$ represents the collection of all nonempty subsets of $Q_{t}^{\prime}$, is called a set-valued homomorphism if, for all $a_{i}, a, b \in$ $Q_{t}(i \in I)$,
(1) $H(a) *_{2} H(b) \subseteq H\left(a *_{1} b\right)$,
(2) $\bigvee_{i \in I} H\left(a_{i}\right) \subseteq H\left(\bigvee_{i \in I} a_{i}\right)$

A set-valued mapping $H: Q_{t} \rightarrow P^{*}\left(Q_{t}^{\prime}\right)$ is called a strong set-valued homomorphism if we replace inclusion by equality in (1) and (2).

From here onwards by SV-Hom, we will mean the setvalued homomorphism. For strong set-valued homomorphism, we will use SSV-Hom. Besides $H$ will mean the map $H: Q_{t} \rightarrow P^{*}\left(Q_{t}^{\prime}\right)$, unless stated otherwise.

\section{Generalized Rough Fuzzy Prime (Primary) Ideals in Quantale}

In this section, we will introduce the generalized rough fuzzy ideal in quantales and resulting properties of such ideals are presented. Now we use the concept from Definition 4 and generalized it in the following.

Definition 10. Let $\left(Q_{t}, *_{1}\right)$ and $\left(Q_{t}^{\prime}, *_{2}\right)$ be two quantales and let $H$ be a SV-Hom. Let $g$ be any fuzzy subset of $Q_{t}^{\prime}$. Then for every $z \in Q_{t}$, one defines

$$
\begin{aligned}
& \underline{H}(g)(z)=\inf _{a \in H(z)} g(a) ; \\
& \bar{H}(g)(z)=\sup _{a \in H(z)} g(a) .
\end{aligned}
$$

Here $\underline{H}(g)$ is the generalized lower approximation and $\bar{H}(g)$ is the generalized upper approximation of the fuzzy subset $g$. The pair $(\underline{H}(g), \bar{H}(g))$ is called generalized rough fuzzy set of $Q_{t}$ if $\underline{H}(g) \neq \bar{H}(g)$.

From here onward by GLA, GUA, and GRF, we will mean generalized lower approximation, generalized upper approximation, and generalized rough fuzzy set, respectively.

Lemma 11. Let $H$ be a SV-Hom. Then for every collection $\left\{g_{i}\right\}_{i \in I} \subseteq \mathscr{F}\left(Q_{t}^{\prime}\right)$,

(1) $\underline{H}\left(\inf _{i \in I} g_{i}\right)=\inf _{i \in I} \underline{H}\left(g_{i}\right)$;

(2) $\bar{H}\left(\sup _{i \in I} g_{i}\right)=\sup _{i \in I} \bar{H}\left(g_{i}\right)$.

Proof. (1) For $x \in Q_{t}$, we have

$$
\begin{aligned}
\underline{H}\left(\inf _{i \in I} g_{i}\right)(x) & =\inf _{a \in H(x)} \inf _{i \in I} g_{i}(a)=\inf _{i \in I} \inf _{a \in H(x)} g_{i}(a) \\
& =\inf _{i \in I} \underline{H}\left(g_{i}\right)(x) .
\end{aligned}
$$

The other item has the similar proof.

Proposition 12. Let $\left(Q_{t}, *_{1}\right)$ and $\left(Q_{t}^{\prime}, *_{2}\right)$ be two quantales and let $H$ be a SV-Hom. Let $g$ be a fuzzy subset of $Q_{t}^{\prime}$. Then for each $\alpha \in[0,1]$, one has the following: 
(1) $\underline{H}(g))_{\alpha}=\underline{H}\left(g_{\alpha}\right)$;

(2) $(\bar{H}(g))_{\alpha}=\bar{H}\left(g_{\alpha}\right)$;

(3) $(\underline{H}(g))_{\alpha^{+}}=\underline{H}\left(g_{\alpha^{+}}\right)$;

(4) $(\bar{H}(g))_{\alpha^{+}}=\bar{H}\left(g_{\alpha^{+}}\right)$.

Proof. (1) Let

$$
\begin{aligned}
z & \in(\underline{H}(g))_{\alpha} \Longleftrightarrow \\
\underline{H}(g)(z) & \geq \alpha \Longleftrightarrow \\
\inf _{a \in H(z)} g(a) & \geq \alpha \Longleftrightarrow \\
g(a) & \geq \alpha \quad \forall a \in H(z) ; \Longleftrightarrow \\
H(z) & \subseteq g_{\alpha} \Longleftrightarrow \\
z & \in \underline{H}\left(g_{\alpha}\right) .
\end{aligned}
$$

Axioms (2), (3), and (4) are similar to the proof of (1).

Definition 13. Let $H$ be a SV-Hom. A fuzzy subset $g$ of the quantale $Q_{t}^{\prime}$ is called a lower [an upper] GRF ideal of $Q_{t}^{\prime}$ if $\underline{H}(g)[\bar{H}(g)]$ is a $f$-ideal of $Q_{t}$. A fuzzy subset $g$ of $Q_{t}^{\prime}$, which is both an upper and a lower GRF ideal of $Q_{t}^{\prime}$, is called GRF ideal of $Q_{t}^{\prime}$.

Now, lower approximations and upper approximations of $f$-ideals of quantales are being studied in the following.

Theorem 14. Let $H$ be a SSV-Hom and let $g$ be a $f$-ideal of $Q_{t}^{\prime}$. Then $\underline{H}(g)$ is a $f$-ideal of $Q_{t}$.

Proof. Since $g$ is a $f$-ideal of $Q_{t}^{\prime}$, by Definition 5, we have $g(a \vee$ $b)=g(a) \wedge g(b)$ and $g(a * b) \geq g(a) \vee g(b) \forall a, b \in Q_{t}^{\prime}$. As $H$ is a SSV-Hom, so $H\left(z_{1} \vee z_{2}\right)=H\left(z_{1}\right) \vee H\left(z_{2}\right), \forall z_{1}, z_{2} \in Q_{t}$.

Therefore,

$$
\underline{H}(g)\left(z_{1} \vee z_{2}\right)=\inf _{e \in H\left(z_{1} \vee z_{2}\right)} g(e)=\inf _{e \in H\left(z_{1}\right) \vee H\left(z_{2}\right)} g(e) .
$$

Since e $\in H\left(z_{1}\right) \vee H\left(z_{2}\right)$, there exist $c_{1} \in H\left(z_{1}\right)$ and $c_{2} \in$ $H\left(z_{2}\right)$ such that $e=c_{1} \vee c_{2}$.

Hence,

$$
\begin{aligned}
\underline{H}(g)\left(z_{1} \vee z_{2}\right) & =\inf _{c_{1} \vee c_{2} \in H\left(z_{1}\right) \vee H\left(z_{2}\right)} g\left(c_{1} \vee c_{2}\right) \\
& =\inf _{c_{1} \in H\left(z_{1}\right), c_{2} \in H\left(z_{2}\right)}\left(g\left(c_{1}\right) \wedge g\left(c_{2}\right)\right) \\
& =\inf \left[\inf _{c_{1} \in H\left(z_{1}\right)} g\left(c_{1}\right), \inf _{c_{2} \in H\left(z_{2}\right)} g\left(c_{2}\right)\right] \\
& =\underline{H}(g)\left(z_{1}\right) \wedge \underline{H}(g)\left(z_{2}\right) .
\end{aligned}
$$

Hence,

$$
\underline{H}(g)\left(z_{1} \vee z_{2}\right)=\underline{H}(g)\left(z_{1}\right) \wedge \underline{H}(g)\left(z_{2}\right) \quad \forall z_{1}, z_{2} \in Q_{t} .
$$

Again since $H$ is a SSV-Hom, hence $H\left(z_{1} *_{1} z_{2}\right)=$ $H\left(z_{1}\right) *_{2} H\left(z_{2}\right) \forall z_{1}, z_{2} \in Q_{t}$.
Thus we have

$$
\begin{aligned}
\underline{H}(g)\left(z_{1} *_{1} z_{2}\right) & =\inf _{e \in H\left(z_{1} *_{1} z_{2}\right)} g(e) \\
& =\inf _{e \in H\left(z_{1}\right) *{ }_{2} H\left(z_{2}\right)} g(e) .
\end{aligned}
$$

Now since $e \in H\left(z_{1}\right) *_{2} H\left(z_{2}\right)$, there exist $c_{1} \in H\left(z_{1}\right)$, $c_{2} \in H\left(z_{2}\right)$ such that $e=c_{1} *_{2} c_{2}$.

Thus,

$$
\begin{aligned}
\underline{H}(g)\left(z_{1} *_{1} z_{2}\right) & =\inf _{c_{1} *_{2} c_{2} \in H\left(z_{1}\right) *_{2} H\left(z_{2}\right)} g\left(c_{1} *_{2} c_{2}\right) \\
& \geq \inf _{c_{1} *_{2} c_{2} \in H\left(z_{1}\right) *_{2} H\left(z_{2}\right)}\left[g\left(c_{1}\right) \vee g\left(c_{2}\right)\right] \\
& =\inf _{c_{1} \in H\left(z_{1}\right), c_{2} \in H\left(z_{2}\right)}\left[g\left(c_{1}\right) \vee g\left(c_{2}\right)\right] \\
& =\sup \left[\inf _{c_{1} \in H\left(z_{1}\right)} g\left(c_{1}\right), \inf _{c_{2} \in H\left(z_{2}\right)} g\left(c_{2}\right)\right] \\
& =\underline{H}(g)\left(z_{1}\right) \vee \underline{H}(g)\left(z_{2}\right) .
\end{aligned}
$$

Hence,

$\underline{H}(g)\left(z_{1} *_{1} z_{2}\right) \geq \underline{H}(g)\left(z_{1}\right) \vee \underline{H}(g)\left(z_{2}\right) \quad \forall z_{1}, z_{2} \in Q_{t}$.

Thus, by (9) and (12), $\underline{H}(g)$ is a $f$-ideal of $Q_{t}$.

Theorem 15. Let $H$ be a SSV-Hom and let $g$ be a $f$-ideal of $Q_{t}^{\prime}$. Then $\bar{H}(g)$ is a $f$-ideal of $Q_{t}$.

Proof. Since $H$ is a SSV-Hom, therefore $H\left(z_{1} \vee z_{2}\right)=H\left(z_{1}\right) \vee$ $H\left(z_{2}\right) \forall z_{1}, z_{2} \in Q_{t}$. Also $g$ is $f$-ideal of $Q_{t}^{\prime}$; hence $g(a \vee b)=$ $g(a) \wedge g(b) \forall a, b \in Q_{t}^{\prime}$.

Consider

$$
\bar{H}(g)\left(z_{1} \vee z_{2}\right)=\sup _{c \in H\left(z_{1} \vee z_{2}\right)} g(c)=\sup _{c \in H\left(z_{1}\right) \vee H\left(z_{2}\right)} g(c) .
$$

For $c \in H\left(z_{1}\right) \vee H\left(z_{2}\right)$, we have $a \in H\left(z_{1}\right)$ and $b \in H\left(z_{2}\right)$ such that $c=a \vee b$.

Hence,

$$
\begin{aligned}
\bar{H}(g)\left(z_{1} \vee z_{2}\right) & =\sup _{a \vee b \in H\left(z_{1}\right) \vee H\left(z_{2}\right)} g(a \vee b) \\
& =\sup _{a \in H\left(z_{1}\right), b \in H\left(z_{2}\right)}[g(a) \wedge g(b)] \\
& =\inf \left[\sup _{a \in H\left(z_{1}\right)} g(a), \sup _{b \in H\left(z_{2}\right)} g(b)\right] \\
& =\bar{H}(g)\left(z_{1}\right) \wedge \bar{H}(g)\left(z_{2}\right) .
\end{aligned}
$$

Thus,

$$
\bar{H}(g)\left(z_{1} \vee z_{2}\right)=\bar{H}(g)\left(z_{1}\right) \wedge \bar{H}(g)\left(z_{2}\right) \quad \forall z_{1}, z_{2} \in Q_{t} .
$$

Now,

$$
\begin{aligned}
\bar{H}(g)\left(z_{1} *_{1} z_{2}\right) & =\sup _{c \in H\left(z_{1} *_{2} z_{2}\right)} g(c) \\
& =\sup _{c \in H\left(z_{1}\right){ }_{2} H\left(z_{2}\right)} g(c) .
\end{aligned}
$$


For $c \in H\left(z_{1}\right) *_{2} H\left(z_{2}\right)$, there exist $a \in H\left(z_{1}\right)$ and $b \in$ $H\left(z_{2}\right)$ such that $c=a *_{2} b$.

Hence,

$$
\begin{aligned}
\bar{H}(g)\left(z_{1} *_{1} z_{2}\right) & =\sup _{a *_{2} b \in H\left(z_{1}\right) *_{2} H\left(z_{2}\right)} g\left(a *_{2} b\right) \\
& \geq \sup _{a \in H\left(z_{1}\right), b \in H\left(z_{2}\right)}[g(a) \vee g(b)] \\
& =\sup \left(\sup _{a \in H\left(z_{1}\right)} g(a), \sup _{b \in H\left(z_{2}\right)} g(b)\right) \\
& =\bar{H}(g)\left(z_{1}\right) \vee \bar{H}(g)\left(z_{2}\right) .
\end{aligned}
$$

Thus,

$$
\bar{H}(g)\left(z_{1} *_{1} z_{2}\right) \geq \bar{H}(g)\left(z_{1}\right) \vee \bar{H}(g)\left(z_{2}\right) \quad \forall z_{1}, z_{2} \in Q_{t} .
$$

Hence by (15) and (18), we have $\bar{H}(g)$ is a $f$-ideal of $Q_{t}$.

By the above two theorems, we have immediately the following corollary.

Corollary 16. Let $H$ be a SSV-Hom and let $g$ be a $f$-ideal of $Q_{t}^{\prime}$. Then $g$ is a GRF ideal of $Q_{t}^{\prime}$.

Proposition 17. Let $H$ be a SSV-Hom. Let $\left\{g_{i}\right\}_{i \in I}$ be a family of $f$-ideals of $Q_{t}^{\prime}$. Then $\underline{H}\left(\inf _{i \in I}\left(g_{i}\right)\right)$ is a $f$-ideal of $Q_{t}$.

Proof. Since every $g_{i}$ is a $f$-ideal for $i \in I$, therefore $\forall x, y \in$ $Q_{t}$,

$$
\begin{aligned}
& \underline{H}\left(\inf _{i \in I} g_{i}\right)(x \vee y)=\left(\inf _{i \in I} \underline{H}\left(g_{i}\right)\right)(x \vee y) \\
& =\inf _{i \in I} \underline{H}\left(g_{i}\right)(x \vee y) \\
& =\inf _{i \in I}\left[\inf \left(\underline{H}\left(g_{i}\right)(x), \underline{H}\left(g_{i}\right)(y)\right)\right] \\
& =\inf \left[\inf _{i \in I} \underline{H}\left(g_{i}\right)(x), \inf _{i \in I} \underline{H}\left(g_{i}\right)(y)\right] \\
& =\inf \left[\underline{H}\left(\inf _{i \in I} g_{i}\right)(x), \underline{H}\left(\inf _{i \in I} g_{i}\right)(y)\right] \\
& =\underline{H}\left(\inf _{i \in I} g_{i}\right)(x) \wedge \underline{H}\left(\inf _{i \in I} g_{i}\right)(y) .
\end{aligned}
$$

Hence,

$$
\begin{aligned}
& \underline{H}\left(\inf _{i \in I} g_{i}\right)(x \vee y) \\
& \quad=\underline{H}\left(\inf _{i \in I} g_{i}\right)(x) \wedge \underline{H}\left(\inf _{i \in I} g_{i}\right)(y) \quad \forall x, y \in Q_{t}, \\
& \underline{H}\left(\inf _{i \in I} g_{i}\right)\left(x *_{1} y\right)=\left(\inf _{i \in I} \underline{H}\left(g_{i}\right)\right)\left(x *_{1} y\right) \\
& \quad=\inf _{i \in I} \underline{H}\left(g_{i}\right)\left(x *_{1} y\right) \\
& \quad \geq \inf _{i \in I}\left[\sup \left(\underline{H}\left(g_{i}\right)(x), \underline{H}\left(g_{i}\right)(y)\right)\right]
\end{aligned}
$$

$$
\begin{aligned}
& =\sup \left[\left(\inf _{i \in I} \underline{H}\left(g_{i}\right)(x)\right),\left(\inf _{i \in I} \underline{H}\left(g_{i}\right)(y)\right)\right] \\
& =\sup \left[\underline{H}\left(\inf _{i \in I} g_{i}\right)(x), \underline{H}\left(\inf _{i \in I} g_{i}\right)(y)\right] \\
& =\underline{H}\left(\inf _{i \in I} g_{i}\right)(x) \vee \underline{H}\left(\inf _{i \in I} g_{i}\right)(y) .
\end{aligned}
$$

Hence,

$$
\begin{aligned}
\underline{H}\left(\inf _{i \in I} g_{i}\right)(x * 1, y) \geq & \underline{H}\left(\inf _{i \in I} g_{i}\right)(x) \\
& \vee \underline{H}\left(\inf _{i \in I} g_{i}\right)(y) \quad \forall x, y \in Q_{t} .
\end{aligned}
$$

Therefore, $\underline{H}\left(\inf _{i \in I} g_{i}\right)$ is a $f$-ideal of $Q_{t}$.

Theorem 18. Let $H$ be a SSV-Hom and let $g$ be a $f$-ideal of $Q_{t}^{\prime}$. Then $\underline{H}(g)$ (respectively, $\bar{H}(g)$ ) is a $f$-ideal of $Q_{t}$ if and only if for each $\alpha \in[0,1], \underline{H}\left(g_{\alpha}\right)$ (respectively, $\bar{H}\left(g_{\alpha}\right)$ ), where $g_{\alpha} \neq \emptyset$, is an ideal of $Q_{t}$.

Proof. Suppose $\underline{H}(g)$ is a $f$-ideal of $Q_{t}$. We need to show that $\underline{H}\left(g_{\alpha}\right)$ is an ideal of $Q_{t}$. Let $z_{1}, z_{2} \in \underline{H}\left(g_{\alpha}\right)$. Then $\underline{H}(g)\left(z_{1}\right) \geq$ $\alpha, \underline{H}(g)\left(z_{2}\right) \geq \alpha$. But since $\underline{H}(g)$ is a $f$-ideal, so $\underline{H}(g)\left(z_{1} \vee\right.$ $\left.z_{2}\right)=\underline{H}(g)\left(z_{1}\right) \wedge \underline{H}(g)\left(z_{2}\right) \geq \alpha$. Hence $z_{1} \vee z_{2} \in \underline{H}\left(g_{\alpha}\right)$. Let $y \in \underline{H}\left(g_{\alpha}\right), z \in Q_{t}$, and $z \leq y$. Then $\underline{H}(g)(z) \geq \underline{H}(g)(y) \geq \alpha$. Thus $z \in \underline{H}\left(g_{\alpha}\right)$. Suppose $y \in \underline{H}\left(g_{\alpha}\right)$ and $\forall z \in Q_{t}$, then $\underline{H}(g)\left(z *_{1} y\right) \geq \underline{H}(g)(z) \vee \underline{H}(g)(y)=\underline{H}(g)(y) \geq \alpha$, and we get $z *_{1} y \in \underline{H}\left(g_{\alpha}\right)$. Similarly, $y *_{1} z \in \underline{H}\left(g_{\alpha}\right)$. Hence, $\underline{H}\left(g_{\alpha}\right)$ is an ideal of $Q_{t}$.

Conversely, assume $\underline{H}\left(g_{\alpha}\right)$ is an ideal of $Q_{t}$. We will show that $\underline{H}(g)$ is a $f$-ideal of $Q_{t}$. For any $z_{1}, z_{2} \in Q_{t}$, let $\alpha=$ $\underline{H}(g)\left(z_{1}\right) \wedge \underline{H}(g)\left(z_{2}\right) \in \operatorname{rang}(\underline{H}(g))$. Then $\underline{H}(g)\left(z_{1}\right) \geq \alpha$ and $\underline{H}(g)\left(z_{2}\right) \geq \alpha$; that is, $z_{1} \in \underline{H}\left(g_{\alpha}\right)$ and $z_{2} \in \underline{H}\left(g_{\alpha}\right)$. Hence, $z_{1} \vee z_{2} \in \underline{H}\left(g_{\alpha}\right)$.

Consider

$\underline{H}(g)\left(z_{1} \vee z_{2}\right)=\inf _{c \in H\left(z_{1} \vee z_{2}\right)} g(c)=\inf _{c \in H\left(z_{1}\right) \vee H\left(z_{2}\right)} g(c)$.

Since $H$ is a SSV-Hom, for $c \in H\left(z_{1}\right) \vee H\left(z_{2}\right)$, there exist $a_{1} \in H\left(z_{1}\right)$ and $a_{2} \in H\left(z_{2}\right)$ such that $c=a_{1} \vee a_{2}$.

Hence we obtain

$$
\begin{aligned}
\underline{H}(g)\left(z_{1} \vee z_{2}\right) & =\inf _{a_{1} \vee a_{2} \in H\left(z_{1}\right) \vee H\left(z_{2}\right)} g\left(a_{1} \vee a_{2}\right) \\
& =\inf _{a_{1} \vee a_{2} \in H\left(z_{1}\right) \vee H\left(z_{2}\right)}\left[g\left(a_{1}\right) \wedge g\left(a_{2}\right)\right] \\
& =\inf _{a_{1} \in H\left(z_{1}\right), a_{2} \in H\left(z_{2}\right)}\left[g\left(a_{1}\right) \wedge g\left(a_{2}\right)\right] \\
& =\inf \left(\inf _{a_{1} \in H\left(z_{1}\right)} g\left(a_{1}\right), \inf _{a_{2} \in H\left(z_{2}\right)} g\left(a_{2}\right)\right) \\
& =\underline{H}(g)\left(z_{1}\right) \wedge \underline{H}(g)\left(z_{2}\right) .
\end{aligned}
$$

So $\underline{H}(g)\left(z_{1} \vee z_{2}\right)=\underline{H}(g)\left(z_{1}\right) \wedge \underline{H}(g)\left(z_{2}\right) \forall z_{1}, z_{2} \in \underline{H}\left(g_{\alpha}\right)$. Now for $z \in \underline{H}\left(g_{\alpha}\right)$ and $\forall y \in Q_{t}$, we obtain $z *_{1} y \in$ $\underline{H}\left(g_{\alpha}\right)$ and $y *_{1} z \in \underline{H}\left(g_{\alpha}\right)$. Hence, $\underline{H}(g)\left(z *_{1} y\right) \geq \alpha$ and 


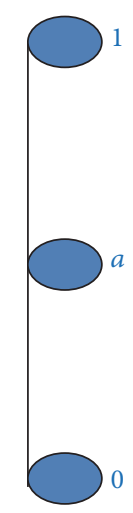

FIgURE 1: Illustration of $Q_{t}$.

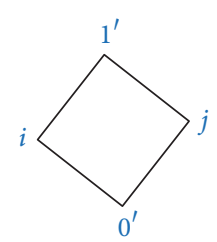

FigURE 2: Illustration of $Q_{t}^{\prime}$.

$\underline{H}(g)(z) \geq \alpha$. If either $\underline{H}(g)(y) \geq \alpha$ or $\underline{H}(g)(y)<\alpha$, in both the cases, $(\underline{H}(g)(x) \vee \underline{H}(g)(y)) \geq \alpha$. We suppose $(\underline{H}(g)(x) \vee$ $\underline{H}(g)(y))=\alpha$. So $\underline{H}(g)\left(x *_{1} y\right) \geq(\underline{H}(g)(x) \vee \underline{H}(g)(y))$. Hence, $\underline{H}(g)$ is a $f$-ideal of $Q_{t}$.

Example 19. Let $\left(Q_{t}, *_{1}\right)$ and $\left(Q_{t}^{\prime}, *_{2}\right)$ be two quantales, where $Q_{t}$ and $Q_{t}^{\prime}$ are depicted in Figures 1 and 2 and the binary operations $*_{1}$ and $*_{2}$ on both the quantales are the same as the meet operation in the lattices $Q_{t}$ and $Q_{t}^{\prime}$ as shown in Tables 1 and 2 .

Let $H$ be a SSV-Hom as defined by $H(0)=\left\{0^{\prime}\right\}, H(a)=$ $\{i, j\}, H(1)=\left\{1^{\prime}\right\}$. Let $g$ be a $f$-ideal of $Q_{t}^{\prime}$ defined by $g=$ $0.9 / 0^{\prime}+0.6 / i+0.7 / j+0.6 / 1^{\prime}$. Then GLA and GUA of the $f-$ ideal $g$ of $Q_{t}^{\prime}$ are as follows: $\underline{H}(g)=0.9 / 0+0.6 / a+0.6 / 1$ and $\bar{H}(g)=0.9 / 0+0.7 / a+0.6 / 1$. It is easily verified that $\underline{H}(g)$ and $\bar{H}(g)$ are $f$-ideals of $Q_{t}$.

Consider $H: Q_{t}^{\prime} \rightarrow P^{*}\left(Q_{t}^{\prime}\right)$ defined by $H\left(0^{\prime}\right)=H(i)=$ $H(j)=\left\{0^{\prime}\right\}$ and $H\left(1^{\prime}\right)=Q_{t}^{\prime}$. Then $H$ is a SV-Hom.

Let $\mu$ be a fuzzy subset of $Q_{t}^{\prime}$ defined by $\mu(x)=\{1$, if $x=$ $0^{\prime}$; 0.7, if $\left.x \neq 0^{\prime}\right\} \forall x \in Q_{t}^{\prime}$. Then $\mu$ is a $f$-ideal of $Q_{t}^{\prime}$. Hence GLA and GUA of $f$-ideal $\mu$ of $Q_{t}^{\prime}$ are $\underline{H}(\mu)=1 / 0^{\prime}+1 / i+1 / j+$ $0.7 / 1^{\prime}$ and $\bar{H}(\mu)=1 / 0^{\prime}+1 / i+1 / j+1 / 1^{\prime}$. It is observed that $\underline{H}(\mu)$ is not a $f$-ideal of $Q_{t}^{\prime}$ and $\bar{H}(\mu)$ is a constant $f$-ideal. Hence it is important to take SSV-Hom.

Definition 20. Let $H$ be a SV-Hom and let $g$ be a fuzzy subset of a quantale $Q_{t}^{\prime}$. Then $g$ is called an upper [a lower] GRF prime ideal of $Q_{t}^{\prime}$ if $\bar{H}(g)[\underline{H}(g)]$ is a $f$-prime ideal of $Q_{t}$. A fuzzy subset $g$ of $Q_{t}^{\prime}$, which is both an upper and a lower GRF prime ideal, is called GRF prime ideal of $Q_{t}^{\prime}$.
TABLE 1: Binary operation $*_{1}$ subject to $Q_{t}$.

\begin{tabular}{llll}
\hline$*_{1}$ & 0 & $a$ & 1 \\
\hline 0 & 0 & 0 & 0 \\
$a$ & 0 & $a$ & $a$ \\
1 & 0 & $a$ & 1 \\
\hline
\end{tabular}

TABLE 2: Binary operation $*_{2}$ subject to $Q_{t}^{\prime}$.

\begin{tabular}{llccc}
\hline$*_{2}$ & $0^{\prime}$ & $i$ & $j$ & $1^{\prime}$ \\
\hline $0^{\prime}$ & $0^{\prime}$ & $0^{\prime}$ & $0^{\prime}$ & $0^{\prime}$ \\
$i$ & $0^{\prime}$ & $i$ & $0^{\prime}$ & $i$ \\
$j$ & $0^{\prime}$ & $0^{\prime}$ & $j$ & $j$ \\
$1^{\prime}$ & $0^{\prime}$ & $i$ & $j$ & $1^{\prime}$ \\
\hline
\end{tabular}

Similarly, we can define upper [lower] GRF semiprime (primary) ideals of quantale. Thus the concept of generalized rough fuzzy ideals of quantales extends the notion of rough fuzzy ideals.

Proposition 21. Let $H$ be a SSV-Hom. If $g$ is a $f$-prime ideal of $Q_{t}^{\prime}$, then $\underline{H}(g)$ is a $f$-prime ideal of $Q_{t}$.

Proof. As $g$ is a $f$-prime ideal of $Q_{t}^{\prime}$, therefore $g\left(c *_{2} b\right)=g(c)$ or $g\left(c *_{2} b\right)=g(b) \forall c, b \in Q_{t}^{\prime}$ and hence, $g$ is a $f$-ideal of $Q_{t}^{\prime}$, so by Theorem $14, \underline{H}(g)$ is a $f$-ideal of $Q_{t}$.

Consider

$$
\begin{aligned}
\underline{H}(g)\left(x_{1} *_{1} y_{1}\right) & =\inf _{e \in H\left(x_{1} *_{1} y_{1}\right)} g(e) \\
& =\inf _{e \in H\left(x_{1}\right) *_{2} H\left(y_{1}\right)} g(e) .
\end{aligned}
$$

Since $H$ is a SSV-Hom, therefore for $e \in H\left(x_{1}\right) *_{2} H\left(y_{1}\right)$ there exist $c \in H\left(x_{1}\right)$ and $b \in H\left(y_{1}\right)$ such that $e=c *_{2} b$.

Hence,

$$
\begin{aligned}
\underline{H}(g)\left(x_{1} *_{1} y_{1}\right) & =\inf _{c *_{2} b \in H\left(x_{1}\right) *_{2} H\left(y_{1}\right)} g\left(c *_{2} b\right) \\
& =\inf _{c \in H\left(x_{1}\right), b \in H\left(y_{1}\right)} g\left(c *_{2} b\right) \\
& =\inf _{c \in H\left(x_{1}\right), b \in H\left(y_{1}\right)}[g(c) \text { or } g(b)] \\
& =\inf _{c \in H\left(x_{1}\right)} g(c) \text { or } \inf _{b \in H\left(y_{1}\right)} g(b) \\
& =\underline{H}(g)\left(x_{1}\right) \text { or } \underline{H}(g)\left(y_{1}\right) .
\end{aligned}
$$

Thus, $\underline{H}(g)\left(x_{1} *_{1} y_{1}\right)=\underline{H}(g)\left(x_{1}\right)$ or $\underline{H}(g)\left(x_{1} *_{1} y_{1}\right)=$ $\underline{H}(g)\left(y_{1}\right) \forall x_{1}, y_{1} \in Q_{t}$. Hence $\underline{H}(g)$ is a $f$-prime ideal of $\mathrm{Q}_{t}$.

Proposition 22. Let $H$ be a SSV-Hom. If $g$ is a $f$-prime ideal of $Q_{t}^{\prime}$, then $\bar{H}(g)$ is a $f$-prime ideal of $Q_{t}$.

Proof. The proof is similar as reported in Proposition 21.

By the above two theorems, we have immediately the following corollary. 
Corollary 23. Let $H$ be a SSV-Hom and let $g$ be a $f$-prime ideal of $Q_{t}^{\prime}$. Then $g$ is a GRF prime ideal of $Q_{t}^{\prime}$.

Theorem 24. Let $H$ be a SSV-Hom and let $\underline{H}(g)$ be a $f$ ideal of $Q_{t}$. Then $\underline{H}(g)$ is a $f$-prime ideal of $Q_{t}$ if and only if $\underline{H}(g)\left(x *_{1} y\right)=\underline{H}(g)(x) \vee \underline{H}(g)(y) \forall x, y \in Q_{t}$.

Proof. Let $\underline{H}(g)$ be a $f$-prime ideal of $Q_{t}$. Then $\underline{H}(g)\left(x *_{1} y\right)=\underline{H}(g)(x)$ or $\underline{H}(g)\left(x *_{1} y\right)=\underline{H}(g)(y)$.

This implies that

$$
\underline{H}(g)\left(x *_{1} y\right) \leq \underline{H}(g)(x) \vee \underline{H}(g)(y) .
$$

As $\underline{H}(g)$ is a $f$-ideal of $Q_{t}$, hence by definition of $f$-ideal, we have

$$
\underline{H}(g)\left(x *{ }_{1} y\right) \geq \underline{H}(g)(x) \vee \underline{H}(g)(y) .
$$

By (26) and (27), we obtain $H(g)(x * 1, y)=H(g)(x) \vee$ $\underline{H}(g)(y)$. Conversely, suppose that $\underline{H}(g)\left(x *_{1} y\right)=\underline{H}(g)(x) \vee$ $\underline{H}(g)(y) \forall x, y \in Q_{t}$. We have to show that $\underline{H}(g)$ is a $f$-prime ideal. As $[0,1]$ is totally ordered, so $\underline{H}(g)(x) \vee$ $\underline{H}(g)(y)=\underline{H}(g)(x)$ or $\underline{H}(g)(x) \vee \underline{H}(g)(y)=\underline{H}(g)(y)$. Hence $\underline{H}(g)\left(x *_{1} y\right)=\underline{H}(g)(x)$ or $\underline{H}(g)\left(x *_{1} y\right)=\underline{H}(g)(y) \forall x, y \in$ $Q_{t}$. This shows that $\underline{H}(g)$ is a $f$-prime ideal of $Q_{t}$.

Theorem 25. Let $H$ be a SSV-Hom and let $g$ be a $f$-prime ideal of $Q_{t}^{\prime}$. Then $\underline{H}(g)$ (respectively, $\left.\bar{H}(g)\right)$ is a $f$-prime ideal of $Q_{t}$ if and only if, for each $\alpha \in[0,1], \underline{H}\left(g_{\alpha}\right)$ (respectively, $\left.\bar{H}\left(g_{\alpha}\right)\right)$, where $g_{\alpha} \neq \emptyset$, is a prime ideal of $Q_{t}$.

Proof. As $g$ is a $f$-prime ideal of $Q_{t}^{\prime}$, therefore $g\left(a *_{2} c\right)=g(a)$ or $g\left(a *_{2} c\right)=g(c) \forall a, c \in Q_{t}^{\prime}$. Suppose $\underline{H}(g)$ is a $f$-prime ideal of $Q_{t}$, then $\underline{H}(g)$ is a $f$-ideal of $Q_{t}$. By Theorem $18, \underline{H}\left(g_{\alpha}\right)$ is an ideal of $Q_{t}$. In order to show that $\underline{H}\left(g_{\alpha}\right)$ is a prime ideal for all $\alpha \in[0,1]$, we have to show that for $a *_{1} c \in \underline{H}\left(g_{\alpha}\right)$ implies that $a \in \underline{H}\left(g_{\alpha}\right)$ or $c \in \underline{H}\left(g_{\alpha}\right)$. Let $a *_{1} c \in \underline{H}\left(g_{\alpha}\right)$. Then $\underline{H}(g)(a)=\underline{H}(g)\left(a *_{1} c\right) \geq \alpha$ or $\underline{H}(g)\left(a *_{1} c\right)=\underline{H}(g)(c) \geq \alpha$. Thus, $a \in \underline{H}\left(g_{\alpha}\right)$ or $c \in \underline{H}\left(g_{\alpha}\right)$. Hence $\underline{H}\left(g_{\alpha}\right)$ is a prime ideal of $Q_{t}$.

Conversely, suppose that $\underline{H}\left(g_{\alpha}\right)$ is a prime ideal of $Q_{t}$, then $\underline{H}\left(g_{\alpha}\right)$ is an ideal of $Q_{t}$. By Theorem 18, $\underline{H}(g)$ is a $f$-ideal of $Q_{t}$.

Consider

$$
\begin{aligned}
\underline{H}(g)\left(x_{2} *_{1} y_{2}\right) & =\inf _{d \in H\left(x_{2} *_{1} y_{2}\right)} g(d) \\
& =\inf _{d \in H\left(x_{2}\right) *_{2} H\left(y_{2}\right)} g(d) .
\end{aligned}
$$

Since $H$ is a SSV-Hom, we have $a \in H(x), c \in H(y)$ such that $d=a *_{2} c$.

Hence,

$$
\begin{aligned}
\underline{H}(g)\left(x_{2} *_{1} y_{2}\right) & =\inf _{a *_{2} c \in H\left(x_{2}\right) *_{2} H\left(y_{2}\right)} g\left(a *_{2} c\right) \\
& =\inf _{a \in H\left(x_{2}\right), c \in H\left(y_{2}\right)} g\left(a *_{2} c\right) \\
& =\inf _{a \in H\left(x_{2}\right), c \in H\left(y_{2}\right)}[g(a) \text { or } g(c)] \\
& =\inf _{a \in H\left(x_{2}\right)} g(a) \text { or } \inf _{c \in H\left(y_{2}\right)} g(c) \\
& =\underline{H}(g)\left(x_{2}\right) \text { or } \underline{H}(g)\left(y_{2}\right) .
\end{aligned}
$$

Therefore $\underline{H}(g)\left(x_{2} *_{1} y_{2}\right)=\underline{H}(g)\left(x_{2}\right)$ or $\underline{H}(g)\left(x_{2} *_{1} y_{2}\right)=$ $\underline{H}(g)\left(y_{2}\right) \forall x_{2}, y_{2} \in Q_{t}$. Hence $\underline{H}(g)$ is a $f$-prime ideal of $Q_{t}$

Theorem 26. Let $H$ be a SSV-Hom and let $g$ be a $f$-semiprime ideal of $Q_{t}^{\prime}$. Then $\underline{H}(g)$ is a $f$-semiprime ideal of $Q_{t}$.

Proof. As $g$ is a $f$-semiprime ideal of $Q_{t}^{\prime}$, therefore $g\left(d^{2}\right)=$ $g(d) \forall d \in Q_{t}^{\prime}$ and $g$ is a $f$-ideal of $Q_{t}^{\prime}$, so by Theorem 14, $\underline{H}(g)$ is a $f$-ideal of $Q_{t}$.

Hence consider

$$
\begin{aligned}
\underline{H}(g)(y) & =\inf _{d \in H(y)} g(d)=\inf _{d \in H(y)} g\left(d^{2}\right) \\
& =\inf _{d *_{2} d \in H(y) *_{2} H(y)} g\left(d^{2}\right) \\
& =\inf _{d *_{2} d \in H\left(y *_{1} y\right)} g\left(d^{2}\right)=\inf _{d^{2} \in H\left(y^{2}\right)} g\left(d^{2}\right) \\
& =\underline{H}(g)\left(y^{2}\right) .
\end{aligned}
$$

Thus $\underline{H}(g)(y)=\underline{H}(g)\left(y^{2}\right) \forall y \in Q_{t}$. Therefore $\underline{H}(g)$ is a $f$-semiprime ideal of $Q_{t}$.

Theorem 27. Let $H$ be a SSV-Hom and let $g$ be a $f$-semiprime ideal of $Q_{t}^{\prime}$. Then $\bar{H}(g)$ is a $f$-semiprime ideal of $Q_{t}$.

Proof. Proof is similar as reported in Theorem 26.

Corollary 28. Let $H$ be a SSV-Hom and let $g$ be a $f$ semiprime ideal of $Q_{t}^{\prime}$. Then $g$ is a GRF semiprime ideal of $Q_{t}^{\prime}$.

Theorem 29. Let $g$ be a $f$-semiprime ideal of $Q_{t}^{\prime}$ and let $H$ be a SSV-Hom. Then $\underline{H}(g)$ (respectively, $\bar{H}(g))$ is a $f$-semiprime ideal of $Q_{t}$ if and only if, for each $\alpha \in[0,1], \underline{H}\left(g_{\alpha}\right)$ (respectively, $\bar{H}\left(g_{\alpha}\right)$ ), where $g_{\alpha} \neq \emptyset$, is a semiprime ideal of $Q_{t}$.

Proof. Suppose $\underline{H}(g)$ is a $f$-semiprime ideal of $Q_{t}$, then $\underline{H}(g)$ is a $f$-ideal of $Q_{t}$. By Theorem 18, $\underline{H}\left(g_{\alpha}\right)$ is an ideal of $Q_{t}$. In order to show that $\underline{H}\left(g_{\alpha}\right)$ is a semiprime ideal $\forall \alpha \in[0,1]$, we have to show that for $a *_{1} a \in \underline{H}\left(g_{\alpha}\right)$ implies $a \in \underline{H}\left(g_{\alpha}\right)$. Let $a *_{1} a \in \underline{H}\left(g_{\alpha}\right)$. Since $\underline{H}(g)$ is a $f$-semiprime ideal, we have $\underline{H}(g)(a)=\underline{H}(g)\left(a *_{1} a\right) \geq \alpha$. Thus, we have $a \in \underline{H}\left(g_{\alpha}\right)$. Hence $\underline{H}\left(g_{\alpha}\right)$ is a semiprime ideal of $Q_{t}$.

Conversely, suppose that $\underline{H}\left(g_{\alpha}\right)$ is a semiprime ideal of $Q_{t}$. Then $\underline{H}\left(g_{\alpha}\right)$ is an ideal of $Q_{t}$. By Theorem $18, \underline{H}(g)$ is a $f$-ideal.

For $\underline{H}(g)$ to be a $f$-semiprime ideal, we have to show that $\underline{H}(g)\left(z *_{1} z\right)=\underline{H}(g)(z) \forall z \in Q_{t}$. As $H$ is a SSV-Hom and $g$ is a $f$-semiprime ideal of $Q_{t}^{\prime}$, consider

$$
\begin{aligned}
\underline{H}(g)(z) & =\inf _{c \in H(z)} g(c)=\inf _{c \in H(z)} g\left(c^{2}\right) \\
& =\inf _{c *_{2} c \in H(z) *_{2} H(z)} g\left(c^{2}\right)=\inf _{c *_{2} c \in H\left(z *_{1} z\right)} g\left(c^{2}\right) \\
& =\inf _{c^{2} \in H\left(z^{2}\right)} g\left(c^{2}\right)=\underline{H}(g)\left(z^{2}\right) .
\end{aligned}
$$

Thus $\underline{H}(g)(z)=\underline{H}(g)\left(z^{2}\right) \forall z \in Q_{t}$. Hence $\underline{H}(g)$ is a $f$ semiprime ideal of $Q_{t}$. 
Example 30. Let $\left(Q_{t}, *_{1}\right)$ and $\left(Q_{t}^{\prime}, *_{2}\right)$ be two quantales, where $Q_{t}$ and $Q_{t}^{\prime}$ are depicted in Figures 1 and 2 and the binary operations $*_{1}$ and $*_{2}$ on both the quantales are the same as the meet operation in the lattices $Q_{t}$ and $Q_{t}^{\prime}$ as shown in Tables 1 and 2 .

Let $H: Q_{t} \rightarrow P^{*}\left(Q_{t}^{\prime}\right)$ be a SSV-Hom as defined in Example 19.

Let $\lambda$ be a fuzzy subset of $Q_{t}^{\prime}$ defined by $\lambda=0.9 / 0^{\prime}+0.6 / i+$ $0.9 / j+0.6 / 1^{\prime}$. Then one can verify that $\lambda$ is a $f$-prime ideal of $Q_{t}^{\prime}$.

Hence GUA and GLA of the $f$-prime ideal $\lambda$ are $\bar{H}(\lambda)=$ $0.9 / 0+0.9 / a+0.6 / 1$ and $\underline{H}(\lambda)=0.9 / 0+0.6 / a+0.6 / 1$. It is observed that $\bar{H}(\lambda)$ and $\underline{H}(\lambda)$ are nonconstant $f$-prime ideals of $Q_{t}$.

Let $g$ be a fuzzy subset of $Q_{t}^{\prime}$ defined by $g(x)=\{1$, if $x=$ $0^{\prime} ; 0.6$, if $\left.x \neq 0^{\prime}\right\} \forall x \in Q_{t}^{\prime}$. Then $g$ is a $f$-semiprime ideal of $Q_{t}^{\prime}$. Hence GLA and GUA of $f$-semiprime ideal $g$ are as follows: $\underline{H}(g)=1 / 0+0.6 / a+0.6 / 1$ and $\bar{H}(g)=1 / 0+0.6 / a+$ $0.6 / 1$. It is clear that $\bar{H}(g)$ and $\underline{H}(g)$ are $f$-semiprime ideals of $Q_{t}$.

Theorem 31. Let $g$ be a $f$-primary ideal of $Q_{t}^{\prime}$ and let $H$ be a $S S V$-Hom. Then $\underline{H}(g)$ is a $f$-primary ideal of $Q_{t}$.

Proof. As $g$ is a $f$-primary ideal of $Q_{t}^{\prime}$, therefore $g\left(a *_{2} b\right)=$ $g(a)$ or $g\left(a *_{2} b\right)=g\left(b^{n}\right) \forall a, b \in Q_{t}^{\prime}$ and hence, $g$ is a $f$-ideal of $Q_{t}^{\prime}$, so by Theorem 14, $\underline{H}(g)$ is $f$-ideal of $Q_{t}$. Since $H$ is given as SSV-Hom, consider

$$
\begin{aligned}
\underline{H}(g)\left(z *_{1} y\right) & =\inf _{d \in H\left(z *_{1} y\right)} g(d) \\
& =\inf _{a *_{2} b \in H(z) *_{2} H(y)} g\left(a *_{2} b\right) \\
& =\inf _{a \in H(z), b \in H(y)} g\left(a *_{2} b\right) \\
& =\inf _{a \in H(z), b \in H(y)}\left[g(a) \text { or } g\left(b^{n}\right)\right] \\
& =\inf _{a \in H(z)} g(a) \text { or } \inf _{b \in H(y)} g\left(b^{n}\right) \\
& =\inf _{a \in H(z)} g(a) \text { or } \inf _{b^{n} \in H\left(y^{n}\right)} g\left(b^{n}\right) \\
& =\underline{H}(g)(z) \text { or } \underline{H}(g)\left(y^{n}\right) .
\end{aligned}
$$

Here $b^{n}=b *_{2} b *_{2}, \ldots, *_{2} b \in H(y) *_{2} H(y) *_{2}, \ldots$, $*_{2} H(y)=H\left(y *_{1} y *_{1} y *_{1}, \ldots, *_{1} y\right)=H\left(y^{n}\right)$ up to $n$ times for some positive integer $n$. Thus $\underline{H}(g)\left(z *_{1} y\right)=\underline{H}(g)(z)$ or $\underline{H}(g)\left(z *_{1} y\right)=\underline{H}(g)\left(y^{n}\right) \forall z, y \in Q_{t}$. Therefore $\underline{H}(g)$ is a $f$ primary ideal of $Q_{t}$.

Theorem 32. Let $g$ be a $f$-primary ideal of $Q_{t}^{\prime}$ and let $H$ be a $S S V$-Hom. Then $\bar{H}(g)$ is a $f$-primary ideal of $Q_{t}$.

Proof. The proof is similar to the proof of Theorem 31.

Theorem 33. Let $H$ be a SSV-Hom and let $g$ be a nonconstant $f$-primary ideal of $Q_{t}^{\prime}$. Then $\underline{H}(g)$ (respectively, $\left.\bar{H}(g)\right)$ is a $f$-primary ideal of $Q_{t}$ if and only if for each $\alpha \in[0,1], \underline{H}\left(g_{\alpha}\right)$ (respectively, $\bar{H}\left(g_{\alpha}\right)$ ), where $g_{\alpha} \neq \emptyset$, is a primary ideal of $Q_{t}$.

\section{Homomorphic Images of Generalized Rough Ideals Based on Quantale Homomorphism}

In this section, we will describe the images of generalized lower and upper approximations by using quantale homomorphism and set-valued homomorphism of quantales.

Definition 34 (see [47]). Let $\left(Q_{t}, *_{1}\right)$ and $\left(Q_{t}^{\prime}, *_{2}\right)$ be two quantales. A map $f: Q_{t} \rightarrow Q_{t}^{\prime}$ is called a quantale homomorphism if

(1) $f\left(a *_{1} b\right)=f(a) *_{2} f(b)$;

(2) $f\left(\bigvee_{i \in I} a_{i}\right)=\bigvee_{i \in I} f\left(a_{i}\right) \forall a, b, a_{i} \in Q_{t}(i \in I)$.

A quantale homomorphism $f: Q_{t} \rightarrow Q_{t}^{\prime}$ is called an epimorphism if $f$ is onto $Q_{t}^{\prime}$ and $f$ is called a monomorphism if $f$ is one-one. If $f$ is bijective, then it is called an isomorphism.

It is clear that if $x \leq y$, then $f(x) \leq f(y)$; that is, $f$ is order-preserving.

Proposition 35. Let $\left(Q_{t}, *_{1}\right)$ and $\left(Q_{t}^{\prime}, *_{2}\right)$ be two quantales, let $f: Q_{t} \rightarrow Q_{t}^{\prime}$ be an epimorphism, and let $H_{2}: Q_{t}^{\prime} \rightarrow P^{*}\left(Q_{t}^{\prime}\right)$ be a SV-Hom. Then one has the following:

(1) If $f$ is one to one and $H_{1}(x)=\left\{y \in Q_{t} \mid f(y) \in\right.$ $\left.H_{2}(f(x))\right\} \forall x \in Q_{t}$, then $H_{1}$ is a SV-Hom from $Q_{t}$ to $P^{*}\left(Q_{t}\right)$.

(2) If $H_{2}$ is a SSV-Hom, then $H_{1}$ is a SSV-Hom.

Proof. (1) First of all, we show that $H_{1}$ is a well-defined mapping. Suppose $x_{1}=x_{2}$, then we have $y_{1} \in H_{1}\left(x_{1}\right) \Leftrightarrow$ $f\left(y_{1}\right) \in H_{2}\left(f\left(x_{1}\right)\right)=H_{2}\left(f\left(x_{2}\right)\right) \Leftrightarrow y_{1} \in H_{1}\left(x_{2}\right)$. Thus we have $H_{1}\left(x_{1}\right)=H_{1}\left(x_{2}\right)$. Now we show that $H_{1}$ is SV-Hom. Suppose $y \in H_{1}\left(x_{1}\right) *_{1} H_{1}\left(x_{2}\right)$, then there exist $a \in H_{1}\left(x_{1}\right)$ and $b \in H_{1}\left(x_{2}\right)$ such that $y=$ $a *{ }_{1} b$. Since $\mathrm{H}_{2}$ is a SV-Hom and $f$ is a quantale homomorphism, then $f(a) *_{2} f(b) \in H_{2}\left(f\left(x_{1}\right)\right) *_{2} H_{2}\left(f\left(x_{2}\right)\right) \subseteq$ $H_{2}\left(f\left(x_{1}\right) *_{2} f\left(x_{2}\right)\right)=H_{2}\left(f\left(x_{1} *_{1} x_{2}\right)\right)$. Therefore, $f\left(a *_{1} b\right)=$ $f(a) *_{2} f(b) \in H_{2}\left(f\left(x_{1} *_{1} x_{2}\right)\right)$. Hence $y=a *_{1} b \in$ $H_{1}\left(x_{1} *_{1} x_{2}\right)$. Thus, we have $H_{1}\left(x_{1}\right) *_{1} H_{1}\left(x_{2}\right) \subseteq H_{1}\left(x_{1} *_{1} x_{2}\right)$. Now we show that $\bigvee_{i \in I} H_{1}\left(x_{i}\right) \subseteq H_{1}\left(\bigvee_{i \in I} x_{i}\right) \forall x_{i} \in Q_{t}(i \in I)$. Let $y \in \bigvee_{i \in I} H_{1}\left(x_{i}\right)$, then there exists $a_{i} \in H_{1}\left(x_{i}\right) \forall i \in I$ such that $y=\bigvee_{i \in I} a_{i}$. Hence $f(y)=f\left(\bigvee_{i \in I} a_{i}\right)=\bigvee_{i \in I} f\left(a_{i}\right) \epsilon$ $\bigvee_{i \in I} H_{2}\left(f\left(x_{i}\right)\right) \subseteq H_{2}\left(\bigvee_{i \in I} f\left(x_{i}\right)\right)=H_{2}\left(f\left(\bigvee_{i \in I} x_{i}\right)\right)$. Finally, $y=\bigvee_{i \in I} a_{i} \in H_{1}\left(\bigvee_{i \in I} x_{i}\right)$. Hence $\bigvee_{i \in I} H_{1}\left(x_{i}\right) \subseteq H_{1}\left(\bigvee_{i \in I} x_{i}\right)$. So, $H_{1}$ is a SV-Hom from $Q_{t}$ to $P^{*}\left(Q_{t}\right)$.

(2) It is similar to part(1).

Theorem 36. Let $f: Q_{t} \rightarrow Q_{t}^{\prime}$ be a quantale isomorphism and let $H_{2}: Q_{t}^{\prime} \rightarrow P^{*}\left(Q_{t}^{\prime}\right)$ be a SV-Hom. Set $H_{1}(m)=\{z \in$ $\left.Q_{t} \mid f(z) \in H_{2}(f(m))\right\} \forall m \in Q_{t}$ and $\forall \emptyset \neq A \subseteq Q_{t}^{\prime}$, then

(1) $f\left(\bar{H}_{1}(A)\right)=\bar{H}_{2}(f(A))$;

(2) $f\left(\underline{H}_{1}(A)\right)=\underline{H}_{2}(f(A))$;

(3) $f(x) \in f\left(\bar{H}_{1}(A)\right) \Leftrightarrow x \in \bar{H}_{1}(A)$. 
Proof. (1) Let $z \in f\left(\bar{H}_{1}(A)\right)$. Then there exists $x \in \bar{H}_{1}(A)$ such that $f(x)=z$. Since $x \in \bar{H}_{1}(A), H_{1}(x) \cap A \neq \emptyset$. Suppose $z^{\prime} \in H_{1}(x) \cap A$, then $f\left(z^{\prime}\right) \in f(A)$, and by the definition of $H_{1}(x)$, we obtain $f\left(z^{\prime}\right) \in H_{2}(f(x))$. Thus, $H_{2}(f(x)) \cap$ $f(A) \neq \emptyset$, and hence $z=f(x) \in \bar{H}_{2}(f(A))$. Thus, we obtain $f\left(\bar{H}_{1}(A)\right) \subseteq \bar{H}_{2}(f(A))$.

Now we take $y \in \bar{H}_{2}(f(A))$, then there is $m \in Q_{t}$ such that $f(m)=y$. Hence $H_{2}(f(m)) \cap f(A) \neq \emptyset$. So there is $z_{1} \in A$ such that $f\left(z_{1}\right) \in f(A)$ and $f\left(z_{1}\right) \in H_{2}(f(m))$. By the definition of $H_{1}(m)$, we have $z_{1} \in H_{1}(m)$. Thus $H_{1}(m) \cap A \neq$ $\emptyset$. This gives $m \in \bar{H}_{1}(A)$. Hence, $y=f(m) \in f\left(\bar{H}_{1}(A)\right)$. Thus $\bar{H}_{2}(f(A)) \subseteq f\left(\bar{H}_{1}(A)\right)$. Finally, we obtain $f\left(\bar{H}_{1}(A)\right)=$ $\bar{H}_{2}(f(A))$.

(2) Suppose $z \in f\left(\underline{H}_{1}(A)\right)$, then there exists $m \in \underline{H}_{1}(A)$ such that $f(m)=z$ and $H_{1}(m) \subseteq A$. Suppose $z^{\prime} \in H_{2}(f(m))$, then there is $n^{\prime} \in Q_{t}$ such that $f\left(n^{\prime}\right)=z^{\prime}$; hence $f\left(n^{\prime}\right) \in$ $H_{2}(f(m))$. Thus $n^{\prime} \in H_{1}(m) \subseteq A$, so $z^{\prime}=f\left(n^{\prime}\right) \in f(A)$. Hence, $H_{2}(f(m)) \subseteq f(A)$. Thus $z=f(m) \in \underline{H}_{2}(f(A))$, so we have $f\left(\underline{H}_{1}(A)\right) \subseteq \underline{H}_{2}(f(A))$.

Now let $y \in \underline{H}_{2}(f(A))$. Then there exists $n \in Q_{t}$ such that $f(n)=y$ and $H_{2}(f(n)) \subseteq f(A)$. Suppose $n^{\prime} \in H_{1}(n)$, then $f\left(n^{\prime}\right) \in H_{2}(f(n)) \subseteq f(A)$ and hence $n^{\prime} \in A$. Thus $H_{1}(n) \subseteq A$ and we obtain $n \in \underline{H}_{1}(A)$. Hence $f(n)=y \in f\left(\underline{H}_{1}(A)\right)$ and thus, $\underline{H}_{2}(f(A)) \subseteq f\left(\underline{H}_{1}(A)\right)$. Hence finally, we have $f\left(\underline{H}_{1}(A)\right)=\underline{H}_{2}(f(A))$.

(3) Let $x \in \bar{H}_{1}(A)$. Then $f(x) \in f\left(\bar{H}_{1}(A)\right)$. Conversely, suppose that $f(x) \in f\left(\bar{H}_{1}(A)\right)$, then there is $y \in \bar{H}_{1}(A)$ such that $f(x)=f(y)$. Since $f$ is ono-one, hence $x=y \in \bar{H}_{1}(A)$.

Remark 37. From Theorem 36(3), it is easily obtained that $f(x) \in f\left(\underline{H}_{1}(A)\right) \Leftrightarrow x \in \underline{H}_{1}(A) \forall \emptyset \neq A \subseteq Q_{t}^{\prime}$.

Theorem 38. Let $f: Q_{t} \rightarrow Q_{t}^{\prime}$ be a surjective quantale homomorphism and let $H_{2}: Q_{t}^{\prime} \rightarrow P^{*}\left(Q_{t}^{\prime}\right)$ be a SV-Hom. Let $H_{1}(x)=\left\{y \in Q_{t} \mid f(y) \in H_{2}(f(x))\right\} \forall x \in Q_{t}$ and $\forall \emptyset \neq A \subseteq Q_{t}^{\prime}$. Then,

(1) $\bar{H}_{1}(A)$ is an ideal of $Q_{t}$ iff $\bar{H}_{2}(f(A))$ is an ideal of $Q_{t}^{\prime}$;

(2) $\bar{H}_{1}(A)$ is a prime ideal of $Q_{t}$ iff $\bar{H}_{2}(f(A))$ is a prime ideal of $Q_{t}^{\prime}$;

(3) $\bar{H}_{1}(A)$ is a semiprime ideal of $Q_{t}$ iff $\bar{H}_{2}(f(A))$ is a semiprime ideal of $Q_{t}^{\prime}$;

(4) $\bar{H}_{1}(A)$ is a primary ideal of $Q_{t}$ iff $\bar{H}_{2}(f(A))$ is a primary ideal of $Q_{t}^{\prime}$.

Proof. (1) Suppose $\bar{H}_{1}(A)$ is an ideal of $Q_{t}$. We show that $\bar{H}_{2}(f(A))$ is an ideal of $Q_{t}^{\prime}$, where $f\left(\bar{H}_{1}(A)\right)=\bar{H}_{2}(f(A))$ by Theorem 36(1).

(i) Let $x, z \in f\left(\bar{H}_{1}(A)\right)$. Then there exists $x_{1}, z_{1} \in \bar{H}_{1}(A)$ such that $f\left(x_{1}\right)=x$ and $f\left(z_{1}\right)=z$. Since $f$ is a surjective quantale homomorphism and $\bar{H}_{1}(A)$ is an ideal of $Q_{t}$, we have $x \vee z=f\left(x_{1}\right) \vee f\left(z_{1}\right)=f\left(x_{1} \vee z_{1}\right) \in f\left(\bar{H}_{1}(A)\right)$. Therefore $x \vee z \in f\left(\bar{H}_{1}(A)\right) \forall x, z \in f\left(\bar{H}_{1}(A)\right)$.

(ii) Let $z \leq x \in f\left(\bar{H}_{1}(A)\right)$. Then we obtain $x_{1} \in \bar{H}_{1}(A)$ and $z_{1} \in Q_{t}$ such that $f\left(x_{1}\right)=x$ and $f\left(z_{1}\right)=z$. Since $f\left(z_{1}\right) \leq f\left(x_{1}\right)$, we have $f\left(x_{1} \vee z_{1}\right)=f\left(x_{1}\right) \vee f\left(z_{1}\right)=f\left(x_{1}\right) \epsilon$ $f\left(\bar{H}_{1}(A)\right)$. But $\bar{H}_{1}(A)$ is a lower set and $z_{1} \leq x_{1} \vee z_{1}$, and we have $z_{1} \in \bar{H}_{1}(A)$. Thus $z=f\left(z_{1}\right) \in f\left(\bar{H}_{1}(A)\right)$.

(iii) Let $x \in f\left(\bar{H}_{1}(A)\right)$ and $z \in Q_{t}^{\prime}$. Then there exist $x_{1} \in$ $\bar{H}_{1}(A)$ and $z_{1} \in Q_{t}$ such that $f\left(x_{1}\right)=x$ and $f\left(z_{1}\right)=z$. Since $\bar{H}_{1}(A)$ is an ideal and $f$ is a quantale homomorphism, we have $x_{1} *_{1} z_{1} \in \bar{H}_{1}(A)$. Hence $x *_{2} z=f\left(x_{1}\right) *_{2} f\left(z_{1}\right)=$ $f\left(x_{1} *_{1} z_{1}\right) \in f\left(\bar{H}_{1}(A)\right)$. In the same way, we have $z *_{2} x \in$ $f\left(\bar{H}_{1}(A)\right)$. Hence, $f\left(\bar{H}_{1}(A)\right)$ is an ideal of $Q_{t}^{\prime}$. But $f\left(\bar{H}_{1}(A)\right)=$ $\bar{H}_{2}(f(A))$. So $\bar{H}_{2}(f(A))$ is an ideal of $Q_{t}^{\prime}$. $Q_{t}^{\prime}$.

Conversely, suppose $f\left(\bar{H}_{1}(A)\right)=\bar{H}_{2}(f(A))$ is an ideal of

(i) Let $z_{1}, z_{2} \in \bar{H}_{1}(A)$. Then $f\left(z_{1}\right), f\left(z_{2}\right) \in f\left(\bar{H}_{1}(A)\right)$. Since $f\left(\bar{H}_{1}(A)\right)$ is directed, $f\left(z_{1} \vee z_{2}\right)=f\left(z_{1}\right) \vee f\left(z_{2}\right) \in$ $f\left(\bar{H}_{1}(A)\right)$. So by Theorem 36(3), we have $z_{1} \vee z_{2} \in \bar{H}_{1}(A)$. Hence $\bar{H}_{1}(A)$ is directed.

(ii) Let $z_{1} \leq z_{2} \in \bar{H}_{1}(A)$. Then $f\left(z_{1}\right) \leq f\left(z_{2}\right) \in$ $f\left(\bar{H}_{1}(A)\right)$. Since $f\left(\bar{H}_{1}(A)\right)$ is a lower set, then $f\left(z_{1}\right) \in$ $f\left(\bar{H}_{1}(A)\right)$. By Theorem 36(3), we obtain $z_{1} \in \bar{H}_{1}(A)$. So $\bar{H}_{1}(A)$ is a lower set.

(iii) Suppose $y^{\prime} \in Q_{t}$ and $y \in \bar{H}_{1}(A)$, then $f\left(y^{\prime}\right) \in Q_{t}^{\prime}$ and $f(y) \in f\left(\bar{H}_{1}(A)\right)$. But $f\left(\bar{H}_{1}(A)\right)$ is an ideal of $Q_{t}^{\prime}$, and we have $f\left(y *_{1} y^{\prime}\right)=f(y) *_{2} f\left(y^{\prime}\right) \in f\left(\bar{H}_{1}(A)\right)$. Thus by Theorem 36(3), we have $y *_{1} y^{\prime} \in \bar{H}_{1}(A)$. Similarly, $y^{\prime} *_{1} y \in$ $\bar{H}_{1}(A)$. So, $\bar{H}_{1}(A)$ is an ideal of $Q_{t}$.

(2) Let $\bar{H}_{1}(A)$ be a prime ideal of $Q_{t}$. Then $\bar{H}_{1}(A)$ is obviously an ideal of $Q_{t}$ and $\bar{H}_{1}(A) \neq Q_{t}$. By part (1), $\bar{H}_{2}(f(A))$ is an ideal of $Q_{t}^{\prime}$. We also have that $\bar{H}_{2}(f(A))=$ $f\left(\bar{H}_{1}(A)\right) \neq Q_{t}^{\prime}$. Now suppose $y_{1}, y_{2} \in Q_{t}^{\prime}$ and $y_{1} *_{2} y_{2} \in$ $\bar{H}_{2}(f(A))$. Since $f$ is surjective, there are $z_{1}, z_{2} \in Q_{t}$ such that $y_{1}=f\left(z_{1}\right), y_{2}=f\left(z_{2}\right)$. Then $f\left(z_{1} *_{1} z_{2}\right)=f\left(z_{1}\right) *_{2} f\left(z_{2}\right)=$ $y_{1} *_{2} y_{2} \in f\left(\bar{H}_{1}(A)\right)$. By Theorem 36(3), we obtain $z_{1} *_{1} z_{2} \epsilon$ $\bar{H}_{1}(A)$. But $\bar{H}_{1}(A)$ is prime, and we have $z_{1} \in \bar{H}_{1}(A)$ or $z_{2} \in \bar{H}_{1}(A)$. Thus $y_{1} \in f\left(\bar{H}_{1}(A)\right)=\bar{H}_{2}(f(A))$ or $y_{2} \in$ $f\left(\bar{H}_{1}(A)\right)=\bar{H}_{2}(f(A))$. So $\bar{H}_{2}(f(A))$ is a prime ideal of $Q_{t}^{\prime}$.

Conversely, let $\bar{H}_{2}(f(A))$ be a prime ideal of $Q_{t}^{\prime}$. Then $\bar{H}_{2}(f(A))$ is an ideal of $Q_{t}^{\prime}$. Since $f\left(\bar{H}_{1}(A)\right)=\bar{H}_{2}(f(A)) \neq$ $Q_{t}^{\prime}$, thus $\bar{H}_{1}(A) \neq Q_{t}$. By part $(1), \bar{H}_{1}(A)$ is an ideal of $Q_{t}$. Now suppose $z_{1}, z_{2} \in Q_{t}$ and $z_{1} *_{1} z_{2} \in \bar{H}_{1}(A)$. So $f\left(z_{1}\right) *_{2} f\left(z_{2}\right)=$ $f\left(z_{1} *_{1} z_{2}\right) \in f\left(\bar{H}_{1}(A)\right)$. Since $f\left(\bar{H}_{1}(A)\right)=\bar{H}_{2}(f(A))$ is prime, we have $f\left(z_{1}\right) \in f\left(\bar{H}_{1}(A)\right)$ or $f\left(z_{2}\right) \in f\left(\bar{H}_{1}(A)\right)$. So by Theorem 36(3), we have $z_{1} \in \bar{H}_{1}(A)$ or $z_{2} \in \bar{H}_{1}(A)$. Thus $\bar{H}_{1}(A)$ is a prime ideal of $Q_{t}$.

The remaining parts (3) and (4) are similar to the proof (2).

Theorem 39. Let $f: Q_{t} \rightarrow Q_{t}^{\prime}$ be a surjective quantale homomorphism and let $H_{2}: Q_{t}^{\prime} \rightarrow P^{*}\left(Q_{t}^{\prime}\right)$ be a SV-Hom. Set $H_{1}(x)=\left\{y \in Q_{t} \mid f(y) \in H_{2}(f(x))\right\} \forall x \in Q_{t}$ and $\forall \emptyset \neq B \subseteq Q_{t}^{\prime}$. Then the following hold:

(1) $\underline{H}_{1}(B)$ is an ideal of $Q_{t}$ iff $\underline{H}_{2}(f(B))$ is an ideal of $Q_{t}^{\prime}$;

(2) $\underline{H}_{1}(B)$ is a prime ideal of $Q_{t}$ iff $\underline{H}_{2}(f(B))$ is a prime ideal of $Q_{t}^{\prime}$; 
(3) $\underline{H}_{1}(B)$ is a semiprime ideal of $Q_{t}$ iff $\underline{H}_{2}(f(B))$ is a semiprime ideal of $Q_{t}^{\prime}$;

(4) $\underline{H}_{1}(B)$ is a primary ideal of $Q_{t}$ iff $\underline{H}_{2}(f(B))$ is a primary ideal of $Q_{t}^{\prime}$.

Proof. The proofs of all the parts can be obtained by Theorem 38 .

\section{Generalized Rough Fuzzy Prime (Primary) Ideals Induced by Quantale Homomorphism}

Theorem 40. Let $f: Q_{t} \rightarrow Q_{t}^{\prime}$ be a surjective quantale homomorphism, let $H_{2}: Q_{t}^{\prime} \rightarrow P^{*}\left(Q_{t}^{\prime}\right)$ be a SV-Hom, and let $\lambda$ be a fuzzy subset of $Q_{t}^{\prime}$. If $H_{1}(x)=\left\{y \in Q_{t} \mid f(y) \in\right.$ $\left.H_{2}(f(x))\right\} \forall x \in Q_{t}$, then

(1) $\bar{H}_{1}(\lambda)$ is a $f$-ideal of $Q_{t}$ iff $\bar{H}_{2}(f(\lambda))$ is a $f$-ideal of $Q_{t}^{\prime}$;

(2) $\bar{H}_{1}(\lambda)$ is a $f$-prime ideal of $Q_{t}$ iff $\bar{H}_{2}(f(\lambda))$ is a $f$ prime ideal of $Q_{t}^{\prime}$;

(3) $\bar{H}_{1}(\lambda)$ is a $f$-semiprime ideal of $Q_{t}$ iff $\bar{H}_{2}(f(\lambda))$ is a $f$-semiprime ideal of $Q_{t}^{\prime}$;

(4) $\bar{H}_{1}(\lambda)$ is a $f$-primary ideal of $Q_{t}$ iff $\bar{H}_{2}(f(\lambda))$ is a $f$ primary ideal of $Q_{t}^{\prime}$.

In the above, $f(\lambda)(y)=\bigvee\left\{\lambda(x) \mid f(x)=y, x \in Q_{t}\right\}$, $y \in Q_{t}^{\prime}$; that is, $f(\lambda)$ is the standard Zadeh image of the fuzzy subset $\lambda$ under the mapping $f$.

Proof. (1) We first point out that, for each $\alpha \in[0,1]$, $(f(\lambda))_{\alpha^{+}}=f\left(\lambda_{\alpha^{+}}\right)$and $\left(\bar{H}_{1}(\lambda)\right)_{\alpha^{+}} \neq \emptyset$ if and only if $\left(\bar{H}_{2}(f(\lambda))\right)_{\alpha^{+}} \neq \emptyset$.

Let $\bar{H}_{1}(\lambda)$ be a $f$-ideal of $Q_{t}$. Then for all $\alpha \in(0,1]$, if $\left(\bar{H}_{2}(f(\lambda))\right)_{\alpha^{+}} \neq \emptyset$, then $\left(\bar{H}_{1}(\lambda)\right)_{\alpha^{+}} \neq \emptyset$. By Theorem 18, we have $\left(\bar{H}_{1}(\lambda)\right)_{\alpha^{+}}$is an ideal of $Q_{t}$. Also by using Proposition 12 , we obtain that $\bar{H}_{1}\left(\lambda_{\alpha^{+}}\right)$is an ideal of $Q_{t}$. Now, by Theorem 38(1), we have that $\left(\bar{H}_{2}(f(\lambda))\right)_{\alpha^{+}}=\bar{H}_{2}(f(\lambda))_{\alpha^{+}}=$ $\bar{H}_{2}\left(f\left(\lambda_{\alpha^{+}}\right)\right)$is an ideal of $Q_{t}^{\prime}$. Thus, by Theorem 18, we have that $\bar{H}_{2}(f(\lambda))$ is a $f$-ideal of $Q_{t}^{\prime}$.

Conversely, suppose $\bar{H}_{2}(f(\lambda))$ is a $f$-ideal of $Q_{t}^{\prime}$. We have that $\left(\bar{H}_{2}(f(\lambda))\right)_{\alpha^{+}}=\bar{H}_{2}(f(\lambda))_{\alpha^{+}}=\bar{H}_{2}\left(f\left(\lambda_{\alpha^{+}}\right)\right)$is an ideal of $Q_{t}^{\prime}$ by utilizing Theorem 18. It is obtained from Theorem 38(1) that $\bar{H}_{1}\left(\lambda_{\alpha^{+}}\right)$is an ideal of $Q_{t}$. Hence by Theorem $18, \bar{H}_{1}(\lambda)$ is a $f$-ideal of $Q_{t}$.

(2) Let $\bar{H}_{1}(\lambda)$ be a $f$-prime ideal of $Q_{t}$. Now for $\bar{H}_{2}(f(\lambda))_{\alpha^{+}} \neq \emptyset$, then $\left(\bar{H}_{1}(\lambda)\right)_{\alpha^{+}} \neq \emptyset$ for any $\alpha \in[0,1]$. Since $\bar{H}_{1}(\lambda)$ is a $f$-prime ideal of $Q_{t}$, then by Theorem 25 , we have that $\left(\bar{H}_{1}(\lambda)\right)_{\alpha^{+}}$is a prime ideal of $Q_{t}$. It is also obtained from Proposition 12 that $\bar{H}_{1}\left(\lambda_{\alpha^{+}}\right)$is a prime ideal of $Q_{t}$. Hence $\left(\bar{H}_{2}(f(\lambda))\right)_{\alpha^{+}}=\bar{H}_{2}(f(\lambda))_{\alpha^{+}}=\bar{H}_{2}\left(f\left(\lambda_{\alpha^{+}}\right)\right)$is a prime ideal of $Q_{t}^{\prime}$, by Theorem 38(2). Thus, by Theorem 25, we have that $\bar{H}_{2}(f(\lambda))$ is a $f$-prime ideal of $Q_{t}^{\prime}$.

Conversely, suppose $\bar{H}_{2}(f(\lambda))$ is a $f$-prime ideal of $Q_{t}^{\prime}$. By Theorem 25, we obtain that $\left(\bar{H}_{2}(f(\lambda))\right)_{\alpha^{+}}=\bar{H}_{2}(f(\lambda))_{\alpha^{+}}=$
$\bar{H}_{2}\left(f\left(\lambda_{\alpha^{+}}\right)\right)$is a prime ideal of $Q_{t}^{\prime}$. Thus it is obtained, from Theorem 38(2), that $\bar{H}_{1}\left(\lambda_{\alpha^{+}}\right)$is a prime ideal of $Q_{t}$. Hence $\bar{H}_{1}(\lambda)$ is a $f$-prime ideal of $Q_{t}$ by Theorem 25 .

Axioms (3) and (4) can be obtained in a similar way.

Theorem 41. Let $f$ be a surjective quantale homomorphism from a quantale $\left(Q_{t}, *_{1}\right)$ onto a quantale $\left(Q_{t}^{\prime}, *_{2}\right)$. Let $H_{2}$ : $Q_{t}^{\prime} \rightarrow P^{*}\left(Q_{t}^{\prime}\right)$ be a SV-Hom and let $\lambda$ be a fuzzy subset of $Q_{t}^{\prime}$. If $H_{1}(x)=\left\{y \in Q_{t} \mid f(y) \in H_{2}(f(x))\right\} \forall x \in Q_{t}$, then

(1) $\underline{H}_{1}(\lambda)$ is a $f$-ideal of $Q_{t}$ iff $\underline{H}_{2}(f(\lambda))$ is a $f$-ideal of $Q_{t}^{\prime}$;

(2) $\underline{H}_{1}(\lambda)$ is a $f$-prime ideal of $Q_{t}$ iff $\underline{H}_{2}(f(\lambda))$ is a $f$ prime ideal of $Q_{t}^{\prime}$;

(3) $\underline{H}_{1}(\lambda)$ is a $f$-semiprime ideal of $Q_{t}$ iff $\underline{H}_{2}(f(\lambda))$ is a $f$-semiprime ideal of $Q_{t}^{\prime}$;

(4) $\underline{H}_{1}(\lambda)$ is a $f$-primary ideal of $Q_{t}$ iff $\underline{H}_{2}(f(\lambda))$ is a $f$ primary ideal of $Q_{t}^{\prime}$.

Proof. The proof is similar as reported in Theorem 40.

\section{Conclusion}

Pure and applied mathematics are two important branches of mathematics and rough set theory has its own importance in both the branches. When we combine rough set theory with algebraic structures, we obtain new interesting results and research topics. These research topics are attracted by computer scientists and mathematicians. Researchers apply roughness into the algebraic system and find interesting algebraic properties of them. The combination of fuzzy set and rough set theory leads to various models. The relations between fuzzy sets, rough sets, and quantale theory have been already considered in [46]. We have examined the generalized rough fuzzy set theory and its properties in quantale.

In the present paper, we substituted a universe set by a quantale and introduced the notions of generalized rough fuzzy prime (semiprime, primary) ideals in quantale. We see that the lower and upper approximations of fuzzy ideals, using SSV-Hom, are fuzzy ideals, respectively. It is also seen that the approximations of fuzzy prime (semiprime, primary) ideals using SSV-Hom are fuzzy prime (semiprime, primary) ideals, respectively. We have discussed the relation between upper (lower) generalized rough fuzzy (prime, semiprime, primary) ideals and upper (lower) generalized rough fuzzy approximations of their homomorphic images.

We believe that in the near future the idea of generalized roughness will be extended to other algebraic structures.

\section{Conflicts of Interest}

There are no conflicts of interest related to this paper.

\section{References}

[1] Z. Pawlak, "Information systems theoretical foundations," Information Systems, vol. 6, no. 3, pp. 205-218, 1981.

[2] Z. Pawlak, "Rough sets," International Journal of Computer \& Information Science, vol. 11, no. 5, pp. 341-356, 1982. 
[3] M. I. Ali, M. Shabir, and S. Tanveer, "Roughness in hemirings," Neural Computing and Applications, vol. 21, no. 1, pp. 171-180, 2012.

[4] R. Biswas and S. Nanda, "Rough groups and rough subgroups," Bulletin of the Polish Academy of Sciences Mathematics, vol. 42, pp. 251-254, 1994.

[5] R. Biswas, "On rough sets and fuzzy rough sets," Bulletin of the Polish Academy of Sciences Mathematics, vol. 42, pp. 345-349, 1994.

[6] R. Biswas, "On rough fuzzy sets," Bulletin of the Polish Academy of Sciences Mathematics, vol. 42, pp. 352-355, 1994.

[7] C. Degang, Z. Wenxiu, D. Yeung, and E. C. C. Tsang, "Rough approximations on a complete completely distributive lattice with applications to generalized rough sets," Information Sciences, vol. 176, no. 13, pp. 1829-1848, 2006.

[8] J. Chen and J. Li, "An application of rough sets to graph theory," Information Sciences, vol. 201, pp. 114-127, 2012.

[9] A. A. Estaji, S. Khodaii, and S. Bahrami, "On rough set and fuzzy sublattice," Information Sciences, vol. 181, no. 18, pp. 3981-3994, 2011.

[10] A. A. Estaji, M. R. Hooshmandasl, and B. Davvaz, "Rough set theory applied to lattice theory," Information Sciences, vol. 200, pp. 108-122, 2012.

[11] V. Leoreanu-Fotea, "Approximations in hypergroups and fuzzy hypergroups," Computers \& Mathematics with Applications, vol. 61, no. 9, pp. 2734-2741, 2011.

[12] J. N. Mordeson, "Rough set theory applied to (fuzzy) ideal theory," Fuzzy Sets and Systems, vol. 121, no. 2, pp. 315-324, 2001.

[13] W. Pedrycz, Granular Computing: Aanalysis and Design of Intelligent System, CRC Press, Boca Raton, Fla, USA, 2013.

[14] S. Rasouli and B. Davvaz, "Roughness in MV-algebras," Information Sciences, vol. 180, no. 5, pp. 737-747, 2010.

[15] Q. Xiao, Q. Li, and X. Zhou, "Rough ideals in Lattices,” Neural Computing and Applications, vol. 21, pp. S245-S253, 2012.

[16] Q. Xiao and Q. Li, "Generalized lower and upper approximations in quantales," Journal of Applied Mathematics, vol. 2012, Article ID 648983, 11 pages, 2012.

[17] Q.-M. Xiao and Z.-L. Zhang, "Rough prime ideals and rough fuzzy prime ideals in semigroups," Information Sciences, vol. 176, no. 6, pp. 725-733, 2006.

[18] X.-Y. Xie and J. Tang, "Fuzzy radicals and prime fuzzy ideals of ordered semigroups," Information Sciences, vol. 178, no. 22, pp. 4357-4374, 2008.

[19] S. Yamak, O. Kazanc1, and B. Davvaz, "Generalized lower and upper approximations in a ring," Information Sciences, vol. 180, no. 9, pp. 1759-1768, 2010.

[20] L. Yang and L. Xu, "Roughness in quantales," Information Sciences, vol. 220, pp. 568-579, 2013.

[21] W. Zhu, "Relationship among basic concepts in covering-based rough sets," Information Sciences, vol. 179, no. 14, pp. 2478-2486, 2009.

[22] W. Zhu and F. Wang, "On three types of covering-based rough sets," IEEE Transactions on Knowledge and Data Engineering, vol. 19, no. 8, pp. 1131-1144, 2007.

[23] L. A. Zadeh, "Fuzzy sets," Information and Control, vol. 8, no. 3, pp. 338-353, 1965.

[24] D. Dubois and H. Prade, "Rough fuzzy sets and fuzzy rough sets," International Journal of General Systems, vol. 17, no. 2-3, pp. 191-209, 1990.
[25] D. Dubois and H. Prade, "Two fold fuzzy sets and rough sets-some issues in knowledge representation," Fuzzy Sets and Systems, vol. 23, no. 1, pp. 3-18, 1987.

[26] A. Nakamura, "Fuzzy rough sets," Note on Multiple-Valued Logic in Japan, vol. 9, no. 8, pp. 1-8, 1988.

[27] S. Nanda and S. Majumdar, "Fuzzy rough sets," Fuzzy Sets and Systems, vol. 45, no. 2, pp. 157-160, 1992.

[28] N. Kuroki and J. N. Mordeson, "Structure of rough sets and rough groups," Journal of Fuzzy Mathematics, vol. 5, no. 1, pp. 183-191, 1997.

[29] N. Kuroki, "Rough ideals in semigroups," Information Sciences, vol. 100, no. 1-4, pp. 139-163, 1997.

[30] B. Davvaz, "Roughness in rings," Information Sciences, vol. 164, no. 1-4, pp. 147-163, 2004.

[31] B. Davvaz, "A short note on algebraic T-rough sets," Information Sciences, vol. 178, no. 16, pp. 3247-3252, 2008.

[32] O. Kazanc1 and B. Davvaz, "On the structure of rough prime (primary) ideals and rough fuzzy prime (primary) ideals in commutative rings," Information Sciences, vol. 178, no. 5, pp. 1343-1354, 2008.

[33] B. Davvaz and M. Mahdavipour, "Roughness in modules," Information Sciences, vol. 176, no. 24, pp. 3658-3674, 2006.

[34] Y. B. Jun, "Roughness of ideals in BCK-algebras," Scientiae Mathematicae Japonicae, vol. 57, no. 1, pp. 165-169, 2003.

[35] J. Zhan, Q. Liu, and B. Davvaz, "A new rough set theory: rough soft hemirings," Journal of Intelligent \& Fuzzy Systems, vol. 28, no. 4, pp. 1687-1697, 2015.

[36] X. Ma, Q. Liu, and J. Zhan, "A survey of decision making methods based on certain hybrid soft set models," Artificial Intelligence Review, vol. 47, no. 4, pp. 507-530, 2017.

[37] X. Ma, J. Zhan, and M. I. Ali, "Applications of a kind of novel Z-soft fuzzy rough ideals to hemirings," Journal of Intelligent \& Fuzzy Systems, vol. 32, no. 3, pp. 2071-2082, 2017.

[38] F. Feng, C. Li, B. Davvaz, and M. I. Ali, "Soft sets combined with fuzzy sets and rough sets: a tentative approach," Soft Computing, vol. 14, no. 9, pp. 899-911, 2010.

[39] D. Meng, X. Zhang, and K. Qin, "Soft rough fuzzy sets and soft fuzzy rough sets," Computers \& Mathematics with Applications, vol. 62, no. 12, pp. 4635-4645, 2011.

[40] J. Zhan and K. Zhu, "A novel soft rough fuzzy set: Z-soft rough fuzzy ideals of hemirings and corresponding decision making," Soft Computing, vol. 21, no. 8, pp. 1923-1936, 2017.

[41] J. Zhan, B. Yu, and V.-E. Fotea, "Characterizations of two kinds of hemirings based on probability spaces," Soft Computing, vol. 20, no. 2, pp. 637-648, 2016.

[42] J. Zhan and K. Zhu, "Reviews on decision making methods based on (fuzzy) soft sets and rough soft sets," Journal of Intelligent \& Fuzzy Systems, vol. 29, no. 3, pp. 1169-1176, 2015.

[43] C. J. Mulvey, "Rendiconti del Circolo Matematico di Palermo," vol. 12, no. 2, pp. 99-104, 1986.

[44] S. Q. Wang and B. Zhao, "Ideals of quantales," Journal of Shaanxi Normal University (Natural Science Edition), vol. 31, no. 4, pp. 710, 2003 (Chinese).

[45] S. Q. Wang and B. Zhao, "Prime ideals and weakly prime ideals of quantales," Fuzzy Systems and Mathematics, vol. 19, no. 1, pp. 78-81, 2005 (Chinese).

[46] Q. Luo and G. Wang, "Roughness and fuzziness in quantales," Information Sciences, vol. 271, pp. 14-30, 2014.

[47] K. I. Rosenthal, Quantales and their applications, Pitman Research Notes in Mathematics, Longman Scientific \& Technical, New York, NY, USA, 1990. 


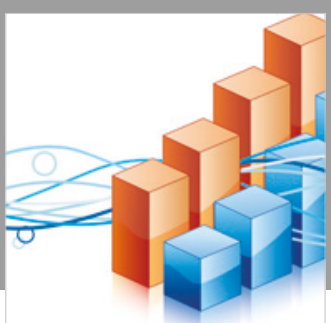

Advances in

Operations Research

\section{-n-m}
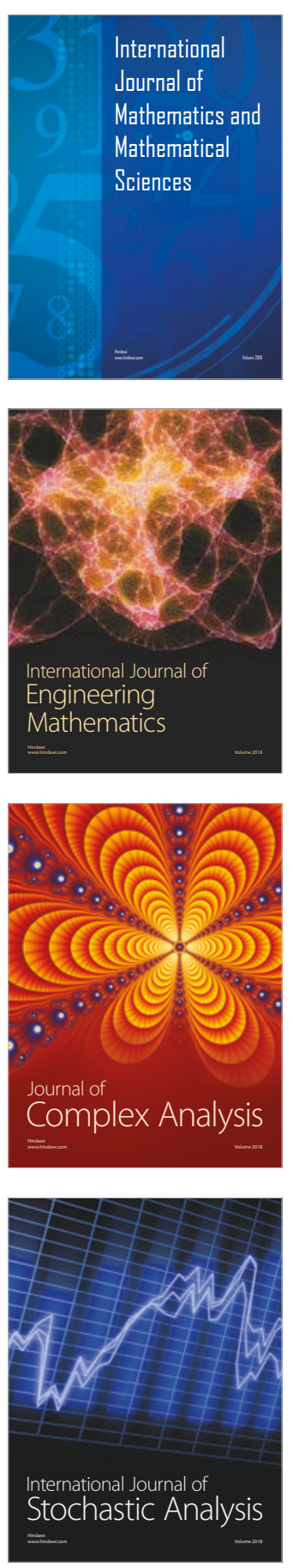
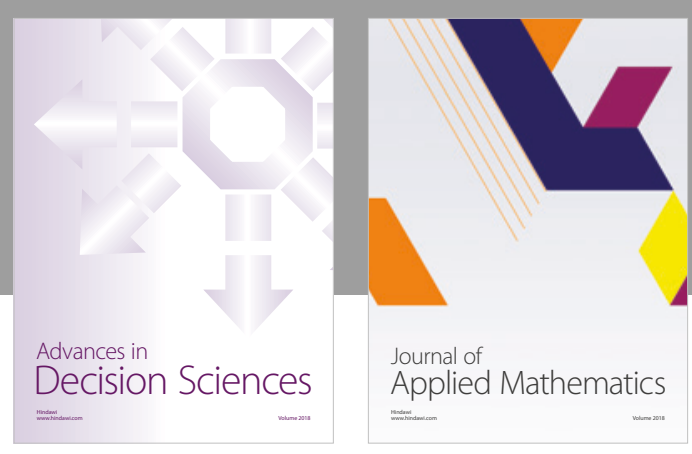

Journal of

Applied Mathematics
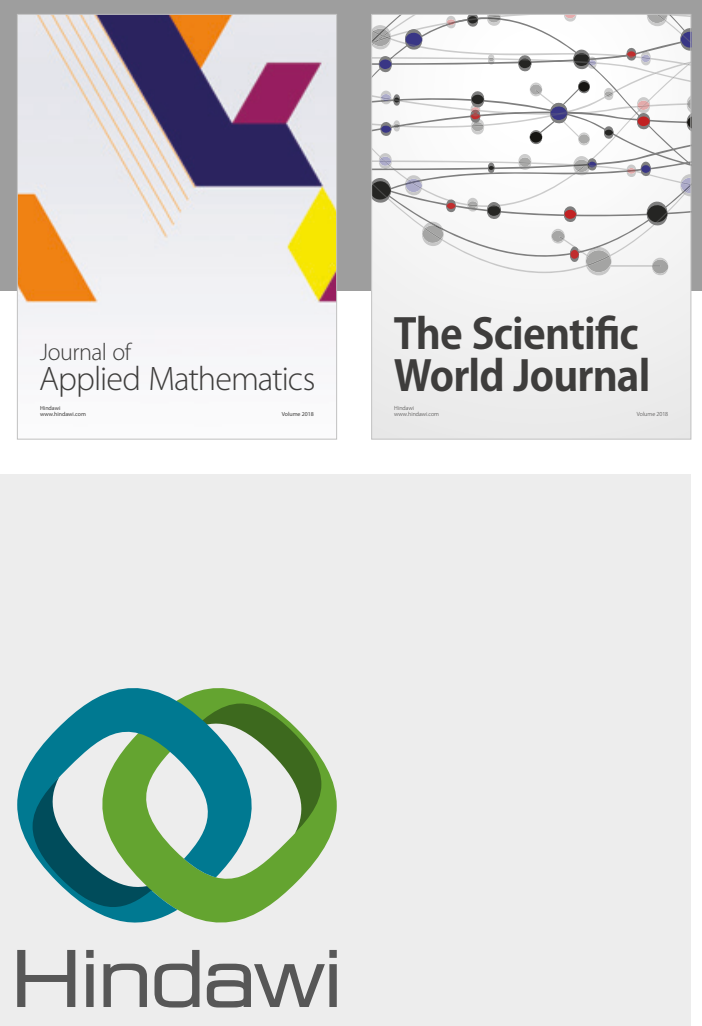

Submit your manuscripts at

www.hindawi.com

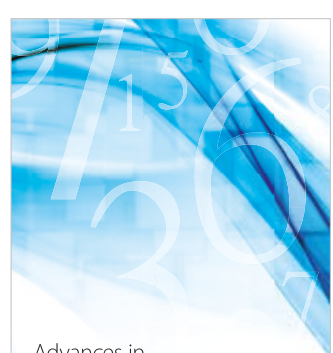

Advances in
Numerical Analysis
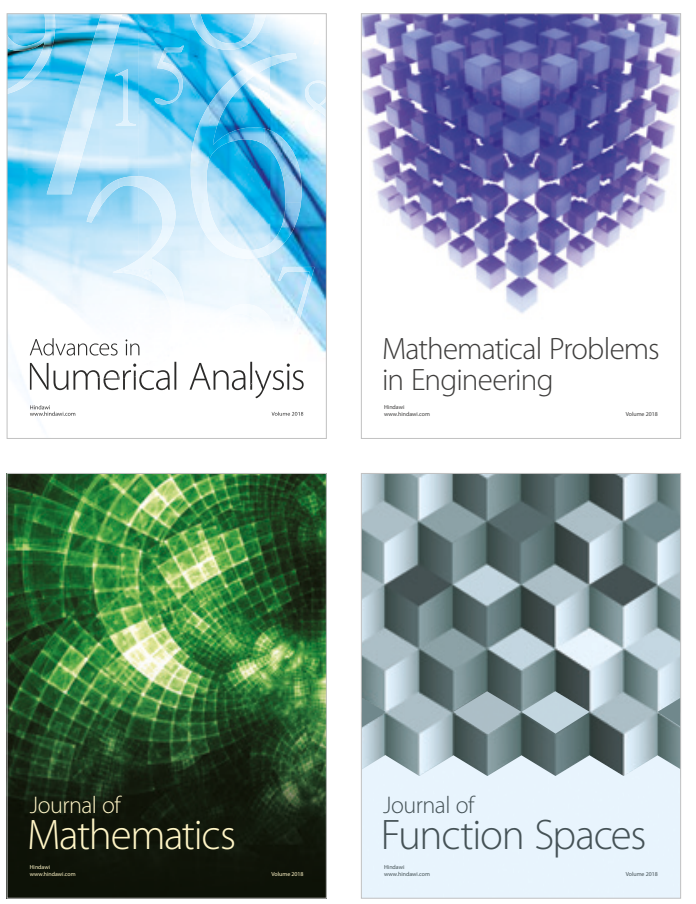

Mathematical Problems in Engineering

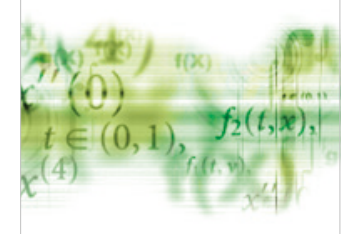

International Journal of

Differential Equations

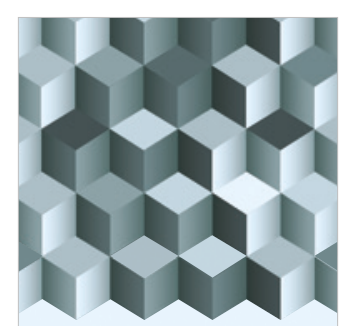

Journal of

Function Spaces

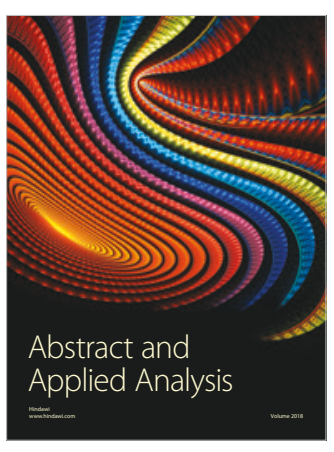

The Scientific

World Journal

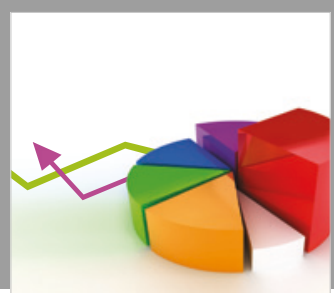

Journal of

Probability and Statistics
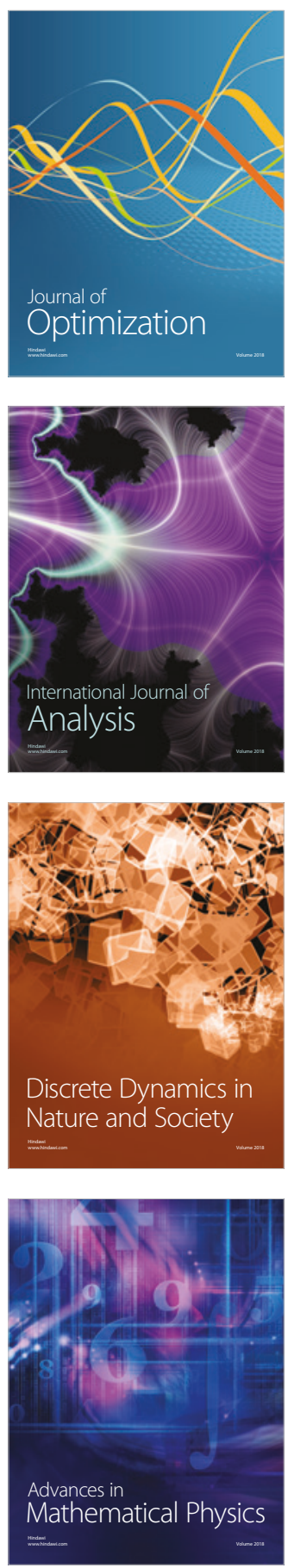